\title{
Deflection by Kinetic Impact: Sensitivity to Asteroid Properties
}

\author{
Megan Bruck Syal ${ }^{\mathrm{a}, *}$, J. Michael Owen ${ }^{\mathrm{a}}$, Paul L. Miller ${ }^{\mathrm{a}}$ \\ ${ }^{a}$ Lawrence Livermore National Laboratory, Livermore, CA 94551, USA
}

\begin{abstract}
Impacting an asteroid with a spacecraft traveling at high speed delivers an impulsive change in velocity to the body. In certain circumstances, this strategy could be used to deflect a hazardous asteroid, moving its orbital path off of an Earth-impacting course. However, the efficacy of momentum delivery to asteroids by hypervelocity impact is sensitive to both the impact conditions (particularly velocity) and specific characteristics of the target asteroid. Here we numerically model asteroid response to kinetic impactors under a wide range of initial conditions, using an Adaptive Smoothed Particle Hydrodynamics code. Impact velocities spanning 1 to $30 \mathrm{~km} / \mathrm{s}$ were investigated, yielding, for a particular set of assumptions about the modeled target material, a power-law dependence consistent with a velocity-scaling exponent of $\mu=0.44$. Target characteristics including equation of state, strength model, porosity, rotational state, and shape were varied, and corresponding changes in asteroid response were documented. The kinetic-impact momentum-multiplication factor, $\beta$, decreases with increasing asteroid cohesion and increasing porosity. Although increased porosity lowers $\beta$, larger porosities result in greater deflection velocities, as a consequence of reduced target masses for asteroids of fixed size. Porosity also lowers disruption risk for kinetic impacts near the threshold of disruption. Including fast $(P=2.5 \mathrm{hr})$ and very fast $(P=100 \mathrm{~s})$ rotation did not significantly alter $\beta$ but did affect the risk of disruption by the impact event. Asteroid shape is found to influence the efficiency of momentum delivery, as local slope conditions can change the orientation of the crater ejecta momentum vector. These results emphasize the need for asteroid characterization studies to bracket the range of target conditions expected at near-Earth asteroids while also highlighting some of the
\end{abstract}

\footnotetext{
*Corresponding author, 1-925-423-0435

Email address: syal1@llnl.gov (Megan Bruck Syal)
} 
principal uncertainties associated with the kinetic-impact deflection strategy.

Keywords: Impact processes; Asteroids; Cratering; Asteroids, dynamics; Asteroids, rotation

\section{1. Introduction}

2

Asteroids posing a threat to Earth may be deflected off of an Earth-impacting trajectory by standoff nuclear bursts (Bruck Syal et al., 2013; Howley et al., 2014) or kinetic impactors (Asphaug et al., 1998; Holsapple and Housen, 2012; Jutzi and Michel, 2014). Among the range of concepts for asteroid threat mitigation, these two methods are considered to be the most technologically mature, as discussed in the report by the National Research Council (2010). While nuclear devices may represent the only viable option to prevent Earth impacts for large asteroids or those detected with little warning time (Dearborn and Miller, 2014), under conditions where a kinetic impactor will be effective, it is the preferred strategy (National Research Council, 2010). Hence, studies which quantify the effectiveness, risks, and uncertainties for the kinetic-impactor method, under a range of initial conditions, are advisable. Full-scale experiments to test the efficacy of kinetic impactors have been and will be rare; these valuable opportunities must be complemented by extensive numerical treatment of the problem. The Deep Impact mission successfully deployed a $370 \mathrm{~kg}$ impactor to remotely excavate the surface of Comet Tempel 1 in 2005 (A'Hearn et al., 2005; Schultz et al., 2007), providing the first demonstration of kinetic-impact technology on a small body. While the large size of Tempel 1 precluded a measurement of the body's change in velocity, future asteroid-defense-focused missions will aim to directly measure the momentum transfer imparted by kinetic impactors. In particular, the AIDA mission, a joint ESA and NASA venture, seeks to provide the first quantitative test of asteroid deflection, using the DART spacecraft to impact the secondary of asteroid Didymos (Cheng et al., 2015).

2 A spacecraft impacting in line with an asteroid's center of mass will transfer all of its momentum, $p_{i}=m_{i} v_{i}$, to the body, changing the asteroid's translational velocity by $\Delta v_{a}=$ $m_{i} v_{i} /\left(m_{a}+m_{i}\right) \approx m_{i} v_{i} / m_{a}$. An additional transfer of momentum is achieved in the cratering process, as material is ejected above escape speed. This additive effect to the momentum 
transfer can be expressed as:

$$
m_{a} \Delta v_{a}=m_{i} v_{i}+m_{e j} v_{e j}=\beta m_{i} v_{i}
$$

where $\beta$ denotes the multiplication factor applied to the impactor's momentum by crater ejecta. The value of $\beta$ is one of the primary uncertainties associated with the use of kinetic impactors. It is known to be dependent on both target material properties and impactor velocity (Asphaug et al., 1998; Holsapple and Housen, 2012; Jutzi and Michel, 2014; Stickle et al., 2015); thus, $\beta$ may vary substantially between different deflection scenarios. Impacts occurring off-axis from the asteroid's center of mass present further complications, as the ejecta flow field is affected by impact angle and the body's rotational state will be altered. The AIDA mission will provide a critical first measurement of $\beta$ for an actual asteroiddeflection event.

There is a great diversity of near-Earth asteroids which may threaten Earth in the future. Numerical study of kinetic-impact deflection - including variability in details such as composition, porosity, strength, internal structure, shape, and rotation - can provide guidelines for kinetic-impactor mission design, including pre-impact reconnaissance of a threatening asteroid. Even in the absence of known near-term threats, information on the sensitivity of various deflection methods to specific asteroid characteristics can inform priorities for future small-body characterization missions. Additionally, numerical simulations, performed for a range of conditions, help define the current limitations of the kinetic-impact approach. Advance knowledge of scenarios where other mitigation methods would be necessary (or provide lower cumulative risk) could speed the response of decision makers in the event of an emergency.

Prior numerical studies of kinetic-impact deflection have focused on planar target geometries (Jutzi and Michel, 2014), or a limited number of cases to simulate the relatively low-speed ( $\sim 6 \mathrm{~km} / \mathrm{s})$ AIDA mission impact (Stickle et al., 2015). Both of these studies used modest impactor masses $(300-400 \mathrm{~kg})$, similar to the planned mass to be delivered by the DART impactor during the AIDA impact event. Additionally, Asphaug et al. (1998) modeled very energetic, disruptive impacts, finding a strong dependence on internal structure for the $\Delta \mathrm{v}$ imparted to the gravitationally bound portion of the asteroid. The present 


\section{2. Numerical approach} impactor.

paper, in contrast, seeks to resolve the limitations and sensitivities of the kinetic-impactor approach within a somewhat broader context, by modeling entire asteroid bodies, using impactor masses representative of the approximate limits posed by current launch vehicle technology $(1000$ - 10,000 kg), and probing sensitivity to a range of asteroid characteristics. Specific asteroid-dependent parameters explored in this work include: equation of state, strength/damage, porosity, rotational state, and shape. In addition, we consider the effects of varying impactor velocity and numerical resolution. Our results are intended to serve as a guide for future simulations incorporating the details of specific asteroids or including additional variations in shape, composition, and internal structure of both the asteroid and

Accurate calculation of the total momentum imparted to an asteroid through hypervelocity impact requires a shock-physics code that can resolve the mass and velocity of particles ejected during the cratering process. Meshless approaches, such as Smoothed Particle Hydrodynamics (SPH) codes (Monaghan, 1992), are particularly well suited to the problem, as mesh entanglement is avoided and tracking ejected mass through large displacements across the problem domain presents fewer complications than many grid-based methods. SPH codes have been used extensively to model impacts at asteroids (see Michel et al. 2015; Asphaug et al. 2015; Jutzi et al. 2015 for reviews of prior work), with Jutzi and Michel (2014) recently applying the method directly to artificial impacts for planetary-defense purposes.

An extension of the SPH method, called Adaptive Smoothed Particle Hydrodynamics (ASPH), generalizes the scalar (isotropic) smoothing length for each SPH particle to a tensor form of smoothing length (Owen et al., 1998; Owen, 2010). This generalization enables ASPH to more accurately define and resolve problems involving anisotropic distortions by allowing the smoothing length scale of each particle to vary with direction; characteristic smoothing lengths in the simulations described here range from $\sim 10-45 \mathrm{~cm}$. For hypervelocity planetary impacts, the tensor generalization is particularly important for capturing the failure of geological materials subjected to large shear stresses. The open-source ASPH code, Spheral, was used to model the kinetic impacts presented in this paper. Unlike many 
standard implementations of SPH, Spheral exactly conserves energy, which presents advantages for demonstrating self convergence in the simulation of kinetic deflection of asteroids (Owen, 2014). All simulations reported here are three dimensional and the number of particles modeled in each problem is substantial $\left(\sim 10^{6}-10^{7}\right)$, requiring a significant number of processors (256-512 cpu) and one to four days of machine time per simulation.

\subsection{Impactor model}

As this paper is primarily concerned with the influence of asteroid characteristics, impactors were modeled simply as uniform aluminum spheres. Future work will examine the effects of impactor shape and mass distribution on deflection results. An extended, lowerdensity source, as opposed to a solid aluminum sphere, will change the impedance matching conditions and the timescale of momentum coupling, which may alter the ejecta velocity distribution and, hence, affect momentum delivery. Quantifying impactor effects will require simulations of sufficient resolution to describe the geometry of different impactors. For specific kinetic-impact missions, resolving the details of the impacting spacecraft may become important, as shown in calculations for the LCROSS impact event (Korycansky et al., 2009). Impact speeds are varied from $1 \mathrm{~km} / \mathrm{s}$ to $30 \mathrm{~km} / \mathrm{s}$, representative of the full range of encounter velocities for spacecraft intercepting the orbits of near-Earth asteroids. While the power-law velocity dependence of $\beta$ can be estimated analytically, using laboratory-scale experimental results (Holsapple and Housen, 2012), numerical simulations provide an opportunity to explore a greater range of velocities than is possible experimentally, at the impact-size regime of interest.

An additional uncertainty associated with the impactor is targeting — in an actual deflection attempt, the impact will, to some degree, be off axis from the asteroid's center of mass. This effect will decrease the $\Delta v$ delivered to the asteroid in at least two ways: some momentum will be partitioned into modifying the asteroid's rotational state, and the obliquity of the impact will influence the direction of the momentum vector associated with the crater ejecta. This second effect is also related to variations in $\Delta v$ introduced by the natural topography of asteroids; an analytical model for perturbations introduced by irregular asteroid shape is presented in Scheeres et al. (2015). These results are further supported by 
112 Spheral calculations using radar-derived shape models, which are briefly discussed in Section

1133.5 and will be presented in detail in a forthcoming paper.

\section{2.2. Asteroid model}

115 Target asteroids were represented by a single, uniform composition $\left(\mathrm{SiO}_{2}\right)$, modeled using 116 a variety of equations of state, including Tillotson (Tillotson, 1962), ANEOS (Thompson and 117 Lauson, 1972; Melosh, 2007), and Livermore Equation of State (LEOS) tables (Fritsch, 2011). 118 While asteroids are not comprised of pure $\mathrm{SiO}_{2}$, this material can be described by a relatively

119

120

121

122

123 well-constrained equation of state (Melosh, 2007; Kraus et al., 2012) and is commonly used for planetary impact modeling. Exploring the effects of variable asteroid composition, including variations introduced by heterogeneous, rubble-pile structures, remains a priority for later studies. A nominal spherical shape was used to model each asteroid, for straightforward comparison across a range of parameters. However, as discussed in Section 3.5, asteroid shape introduces non-trivial changes to the imparted deflection velocity and should be accounted for in uncertainty assessments.

Most asteroids for which bulk-density information is available are believed to contain significant porosity, in the form of microporosity (at the grain level), macroporosity (larger voids between boulders and rubble-pile fragments), or some combination of the two (Britt and Consolmagno, 2000; Britt et al., 2002; Lindsay et al., 2015). Here we focus on microporosity; investigating the effects of macroporous structures is reserved for future calculations. Spheral uses a strain-based approach, defined in Wünnemann et al. (2006), to include asteroid microporosity. The strain-based porosity method, termed the $\epsilon-\alpha$ model, contains an elastic regime, an exponential-compaction regime, and a power-law compaction regime; only the initial porosity $\phi$, elastic-plastic transition strain $\epsilon_{e}$, power law transition strain $\epsilon_{X}$, and exponential compaction rate $\kappa$ need to be defined (Wünnemann et al., 2006). Values used for these parameters are listed in Table 1.

Asteroid strength was included using either a von Mises yield criterion (constant strength) or a pressure-dependent model for strength:

$$
Y_{i}=Y_{0}+\frac{\mu_{i} P}{1+\mu_{i} P /\left(Y_{M}-Y_{0}\right)}
$$


where $Y_{0}$ is shear strength at zero pressure (cohesion), $\mu_{i}$ is the coefficient of internal friction, and $Y_{M}$ is the von Mises plastic limit of the material (Lundborg, 1968; Collins et al., 2004). Recent work highlights the importance of including pressure-dependent constitutive models (i.e., friction) when calculating the outcomes of asteroid collisions (Jutzi, 2015); hence, most simulations reported here used a pressure-dependent strength model. As direct measurements of asteroid strength are not yet available, a range of values for asteroid cohesion, $Y_{0}$ (yield strength at zero pressure), were explored: $Y_{0}=1 \mathrm{kPa}-100 \mathrm{MPa}$. The lower end of this range corresponds to typical values for weak soils (e.g., lunar regolith), while the upper end is representative of strong, competent rock. However, strength is size-dependent; while 1-cm samples of Allende meteorite crush at 25-50 MPa, these strengths decrease to $~ 1 \mathrm{MPa}$ when extrapolated to 5-m sample sizes (Cotto-Figueroa et al., 2015). In the absence of available yield-strength data on asteroidal materials at high pressures, a constant value of $Y_{m}=1.5$ GPa was assumed (Collins et al., 2004). A value of $\mu_{i}=1.2$, consistent with the $50^{\circ}$ angle of internal friction determined for lunar soil samples (Mitchell et al., 1972), was used for all simulations.

Meteorites can provide clues to the strength properties of asteroids; however, consideration should be given to: (1) scaling of strength from laboratory-scale measurements to bodies hundreds of meters in scale, including possible micro- and macro-structural differences between solid meteorite specimens and asteroids (many of which may have rubble-pile structures); (2) whether available meteorite samples are representative of near-Earth asteroids (samples are biased towards compositions likely to survive atmospheric entry and be recognized by meteorite hunters); and (3) changes in physical properties introduced by the reentry process. Regardless, strength measurements from meteorites, particularly at high pressures and a variety of strain rates, are valuable for asteroid impact studies. While there has been little data published on the mechanical properties of meteorites (Petrovic, 2001), interest in planetary defense applications has spurred new efforts. Measurements of an L5 chondrite by Kimberley and Ramesh (2011) demonstrated increased compressive strength from $\sim 50 \mathrm{MPa}$ to $\sim 200 \mathrm{MPa}$ with increasing strain rate. Efforts are underway to obtain both compressive and tensile strength data from a variety of chondrite samples at a wide range of strain rates (Cotto-Figueroa et al., 2015; Sears et al., 2015). 
Damage, which is accumulated through strain-rate-dependent, dynamic-fracture mechanisms, reduces the strength of rocky materials; therefore, asteroid strength is coupled to a 171 damage model, which tracks the strain history of each particle and calculates the extent of 172 damage sustained throughout the impact event. The damage model employs a statistical 173 representation of flaw-activation strains, initially described by Grady and Kipp (1980) and 174 later applied to SPH calculations by Benz and Asphaug (1994, 1995). These (and subse175 quent) SPH implementations of the Grady-Kipp fracture model used a scalar representation 176 for damage, $D$; here we use a similar approach, with the damage variable generalized to 177 a tensor form, $D_{\alpha \beta}$ for each ASPH node (Owen et al., 2015). The eigenvalues of $D_{\alpha \beta}$ are 178 bounded in the range $[0,1]$, and represent the fraction of damage in the direction of their as-

179 180 181 sociated eigenvector. $D_{\alpha \beta}$ represents the history of the damage (including directionality) for each ASPH node, and follows that node throughout the evolution of the simulation. Weibull parameters used to describe the initial flaw distributions are listed in Table 1.

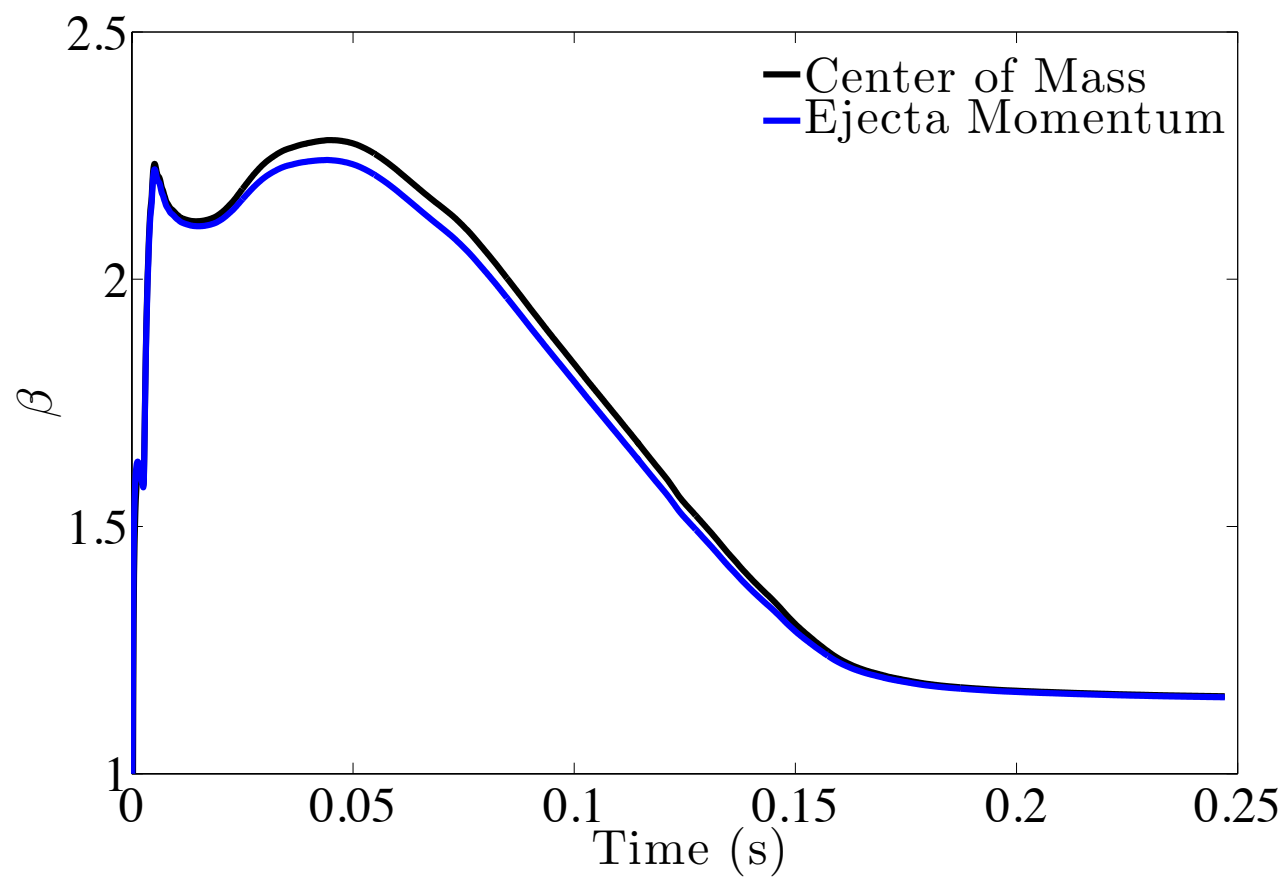

Figure 1: Comparison of two different methods for calculating the momentum multiplication factor, $\beta$ : tracking the asteroid's center of mass (black) and summing the momentum of ejecta exceeding escape velocity (blue). The two methods yield very similar results throughout the simulated time; final values for $\beta$ are nearly identical ( $<0.2 \%$ difference). Results are from run 21 (Table 2). 


\section{3. Results}

\subsection{Calculation of $\beta$}

Calculation of the total momentum delivered to an asteroid is carried out using two separate methods, as a check on consistency. The first method sums the vertically-directed momentum of ejecta exceeding escape velocity (e.g., $v_{e s c}=4.71 \mathrm{~cm} / \mathrm{s}$ for a $100-\mathrm{m}$ asteroid with $40 \%$ porosity). The time evolution of this momentum is followed until the impulse converges (typically, 0.1-0.5 s after impact). For the asteroid sizes considered here (50-100 $\mathrm{m})$, kinetic energy lost to material that is escaping from the asteroid's gravity is negligible. For example, accounting for the change in final velocity from gravitational effects, $v_{i n f}^{2}=$ $v_{e j}^{2}-v_{e s c}^{2}$, for a $5 \mathrm{~km} / \mathrm{s}$ impact into a $40 \%$ porous, $100-\mathrm{m}$ asteroid, reduces the calculated $\beta$ by $<0.1 \%$. As a result, we report $\beta$ values without including energy losses to gravity.

The second $\beta$ calculation method tracks the center-of-mass velocity of the asteroid after impact, by summing the momentum of all material remaining below escape velocity. This method requires modeling the entire asteroid, as was done for nearly all calculations in this study but, depending on asteroid size and desired resolution, may not always be possible. Direct comparison of the two $\beta$ calculation methods shows very good agreement, as shown for a test case in Figure 1. This provides enhanced confidence in using either approach to calculate momentum transfer. Note that $\beta$ decreases from initially larger values during the impact event, owing to the early-time, instantaneous velocities of material that remains bound to the asteroid. The full list of simulation results is provided in Table 2.

\subsection{Equation of State}

Perhaps the most fundamental material property choice is which equation of state should be used to represent the target asteroid. The time evolution of the momentum-transfer multiplier, $\beta$, for $10 \mathrm{~km} / \mathrm{s}$ impacts into $50 \mathrm{~m}$-diameter asteroids, modeled using three different $\mathrm{SiO}_{2}$ equations of state, is shown in Figure 2. Strength and damage models were not included for the purposes of this comparison. Strength (as evidenced in the results for Tillotson with strength shown in Figure 2 and discussed in detail in Section 3.2) can significantly reduce $\beta$. Calculated values for $\beta$, measured by either the momentum of ejecta exceeding escape velocity or the asteroid's center-of-mass velocity, converge within $\sim 0.2$ seconds post-impact. 


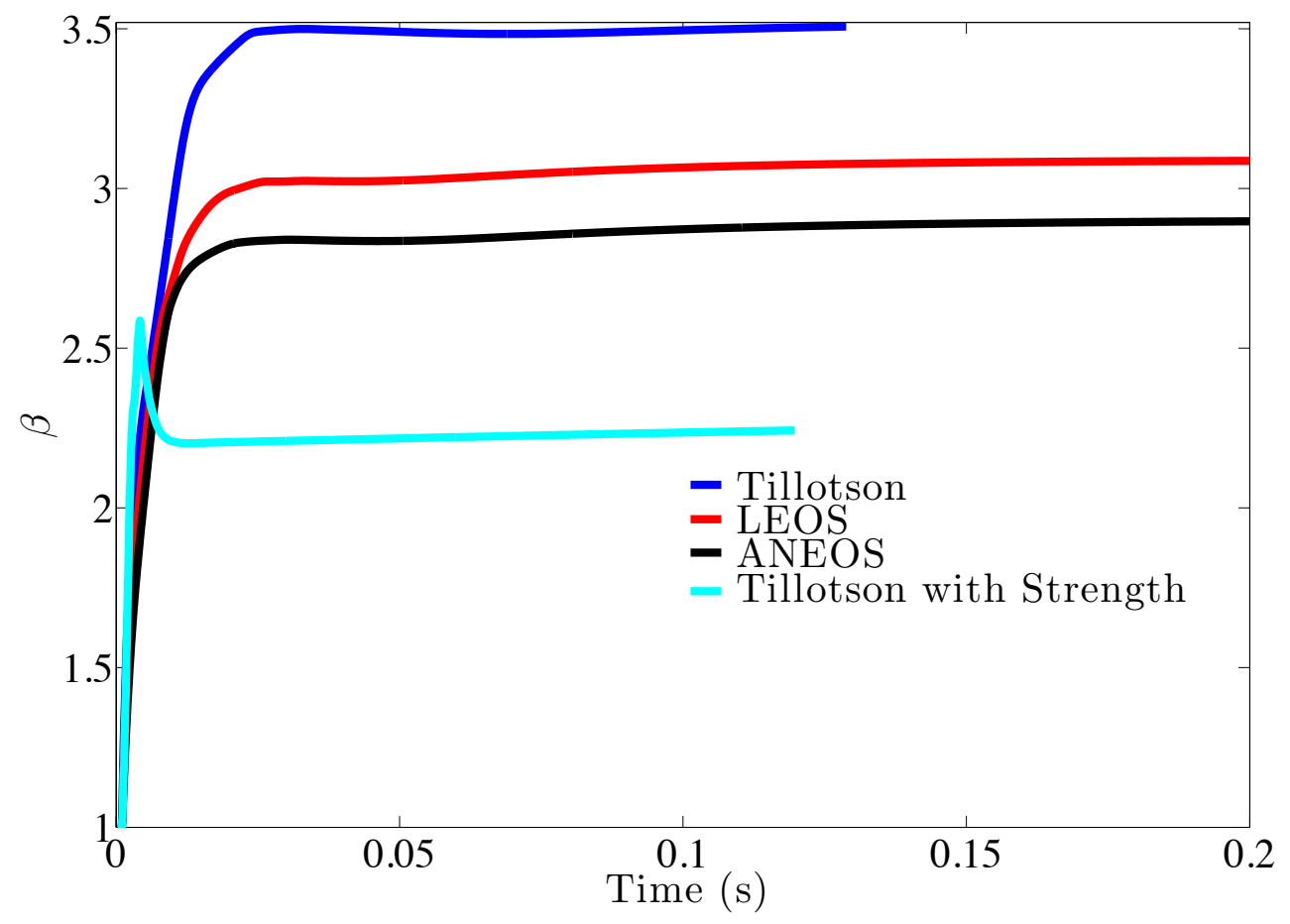

Figure 2: Momentum multiplication factor, $\beta$, plotted as a function of time for kinetic-impact simulations, using three different $\mathrm{SiO}_{2}$ equations of state: Tillotson (Tillotson, 1962), LEOS (Fritsch, 2011), and ANEOS (Thompson and Lauson, 1972; Melosh, 2007). To simplify comparison, no strength or damage models were initially included (blue, red, black lines). Including strength (cyan line) reduces $\beta$ significantly. Momentum delivery converges to a final value within the first $\sim 0.2 \mathrm{~s}$. While Tillotson is a simpler analytic equation of state and useful under a range of lower-velocity impact conditions, LEOS and ANEOS capture melting and vaporization processes more accurately. ANEOS is used for most impact simulations in this paper. Figure shows results from runs 1-4 (Table 2).

The Tillotson model is among the simplest analytic equations of state, and it predicts 212 somewhat larger $\beta$ values than the more sophisticated LEOS (Fritsch, 2011) and ANEOS 213 models. The LEOS tabular equation of state and the ANEOS semi-analytical equation of 214 state include accurate treatment of melt and vapor transitions, unlike Tillotson; lower values 215 of $\beta$ for these cases are attributed to more impact energy being partitioned into melting 216 and vaporizing target material, with less energy partitioned into crater ejecta. The ANEOS 217 package, which tracks regions of mixed melt and vapor in a thermodynamically consistent 218 way (Thompson and Lauson, 1972; Benz et al., 1989; Melosh, 2007) and is already used 219 extensively within the planetary-impacts community, is used for the bulk of simulations 
reported in this paper.

221

222

223

224

225

226

227

228

229

230

231

232

233

234

235

236

237

238

239

240

241

242

243

244

245

246

$247 Y_{0}=1 \mathrm{kPa}$ and $Y_{0}=100 \mathrm{MPa}$. In the case of weaker material $\left(Y_{0}=1 \mathrm{kPa}\right)$, momentum 248 transfer decreases sharply between the nonporous and slightly porous $(\phi=0.05)$ cases and

\subsection{Strength and Damage Model}

The presence of strength, coupled with a damage model, results in the dissipation of impact energy by additional mechanisms, as material is plastically deformed and existing flaws in the rock are activated, leading to crack growth, failure, and fragmentation. Figure 3 compares the $\beta$ values for asteroids with $40 \%$ porosity but with different values of cohesion, $Y_{0}$, for $10 \mathrm{~km} / \mathrm{s}$ impacts. A pressure-dependent strength model was used (Equation 2) for all but one of the cases, where a simple von Mises yield criterion (constant strength) was implemented. The difference between these two strength models is small: $\beta$ is calculated to be $<2 \%$ greater for the constant strength case than for the pressure-dependent model (for $Y_{0}=100 \mathrm{MPa}$ ). Prior calculations of $\beta$ by Jutzi and Michel (2014) used pressure-dependent strength for all simulations.

Low-cohesion asteroids result in larger values of $\beta$; however, there is little difference between $1 \mathrm{kPa}$ (very weak soils), $10 \mathrm{kPa}$ (weak soils), and $100 \mathrm{kPa}$ (weak rocks). This range brackets the size-dependent strength values for all but the strongest meteorite types. At somewhat larger values of cohesion (>1 MPa), $\beta$ declines more noticeably. The strengthless example produces a significantly larger momentum-transfer efficiency $(\beta \sim 5.5)$ than the lowest-cohesion case. Assuming that powdery lunar regolith represents a minimum value of cohesion for an airless silicate asteroid, a value of $Y_{0}=1 \mathrm{kPa}$ is used for many of the comparative simulations, as it represents an approximate lower limit of the shear strength at zero pressure for an asteroid and can be viewed as an "upper limit" for enhancing $\beta$ in a deflection scenario.

\subsection{Porosity}

Asteroid microporosity decreases the augmentation of momentum transfer from a kinetic impactor, as impact energy is consumed in the crushing of pore spaces, leaving less energy available for the ejection of target material. Figure 4 illustrates the effect of microporosity on the momentum-enhancement factor, $\beta$, using two different values for asteroid cohesion: 


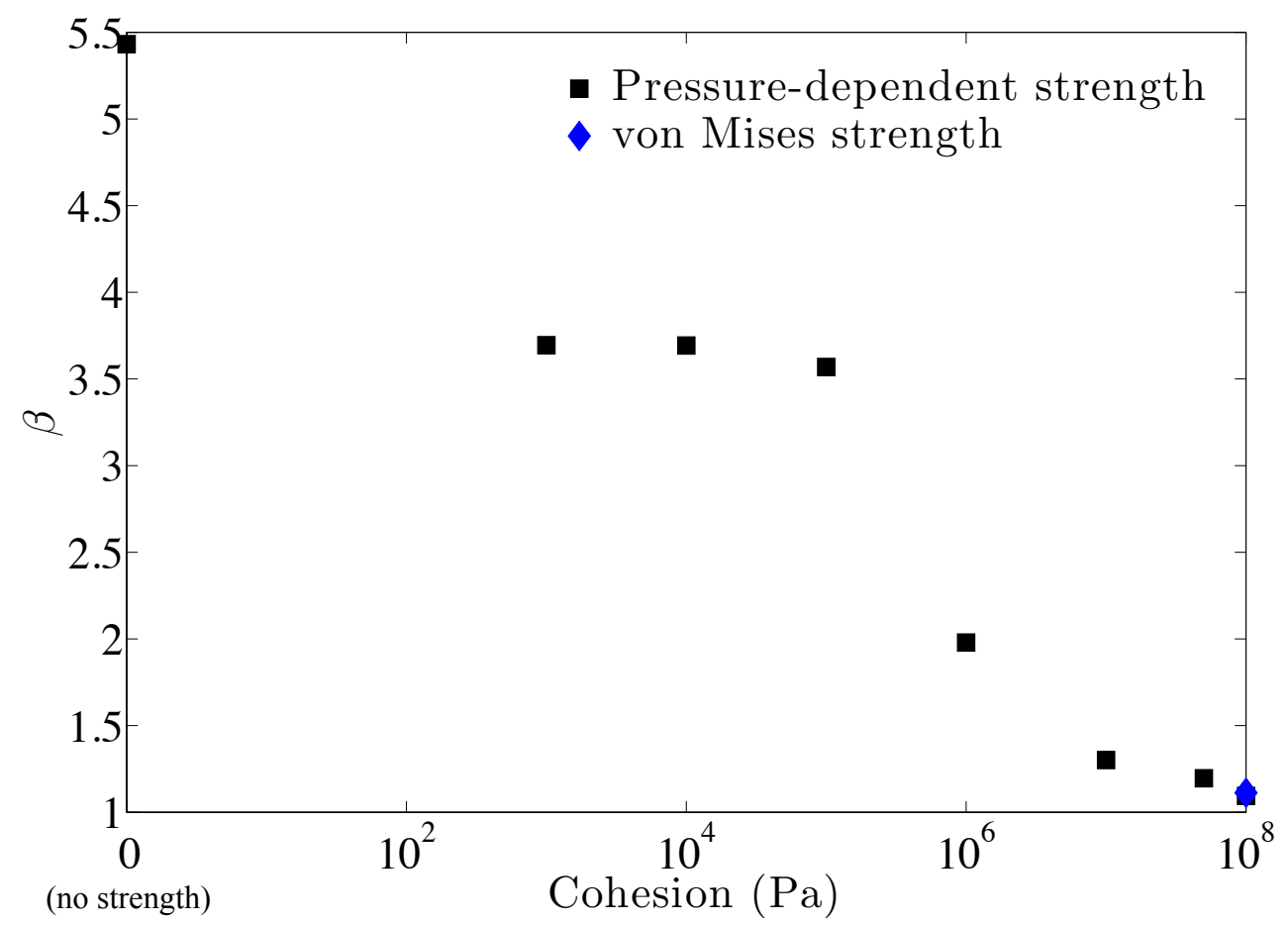

Figure 3: Increasing values of cohesion for asteroid material decreases the momentum enhancement factor, $\beta$, for kinetic deflections. Modeling the asteroid as strengthless results in a significant over estimate: $\beta \sim 5.5$. Cohesion values representative of weak soils $(1 \mathrm{kPa})$ to weak rocks $(100 \mathrm{kPa})$ produce similar deflection velocities, while somewhat larger values of cohesion (1-100 MPa, representative of stronger rocks), lead to increasingly smaller values for $\beta$. Use of a constant (von Mises criterion) strength for the $100 \mathrm{MPa}$ case produced a slightly larger $\beta$ value than the pressure-dependent strength model; the latter is considered more accurate and used in the majority of the simulations. Data from runs 5-13 (Table 2).

then decreases more gradually with increasing microporosity. Porosity effects are more subtle for the stronger material $\left(Y_{0}=100 \mathrm{MPa}\right)$.

However, asteroid porosity also reduces total target mass for a fixed-size asteroid, lowering the momentum necessary to achieve a desired change in velocity. Prior to a deflection mission, a likely scenario may include information on an asteroid's surface composition and diameter from remote sensing data but little or no direct information on mass or bulk density. In this situation, the uncertainty in asteroid density/porosity will result in two competing effects: larger porosities will reduce $\beta$ but a corresponding lower asteroid mass will also lead to a larger $\Delta v$ for a given momentum impulse. Figure 5 illustrates how the sum of both effects actually results in larger total $\Delta v$ for more porous asteroids. Greater asteroid porosity is thus 


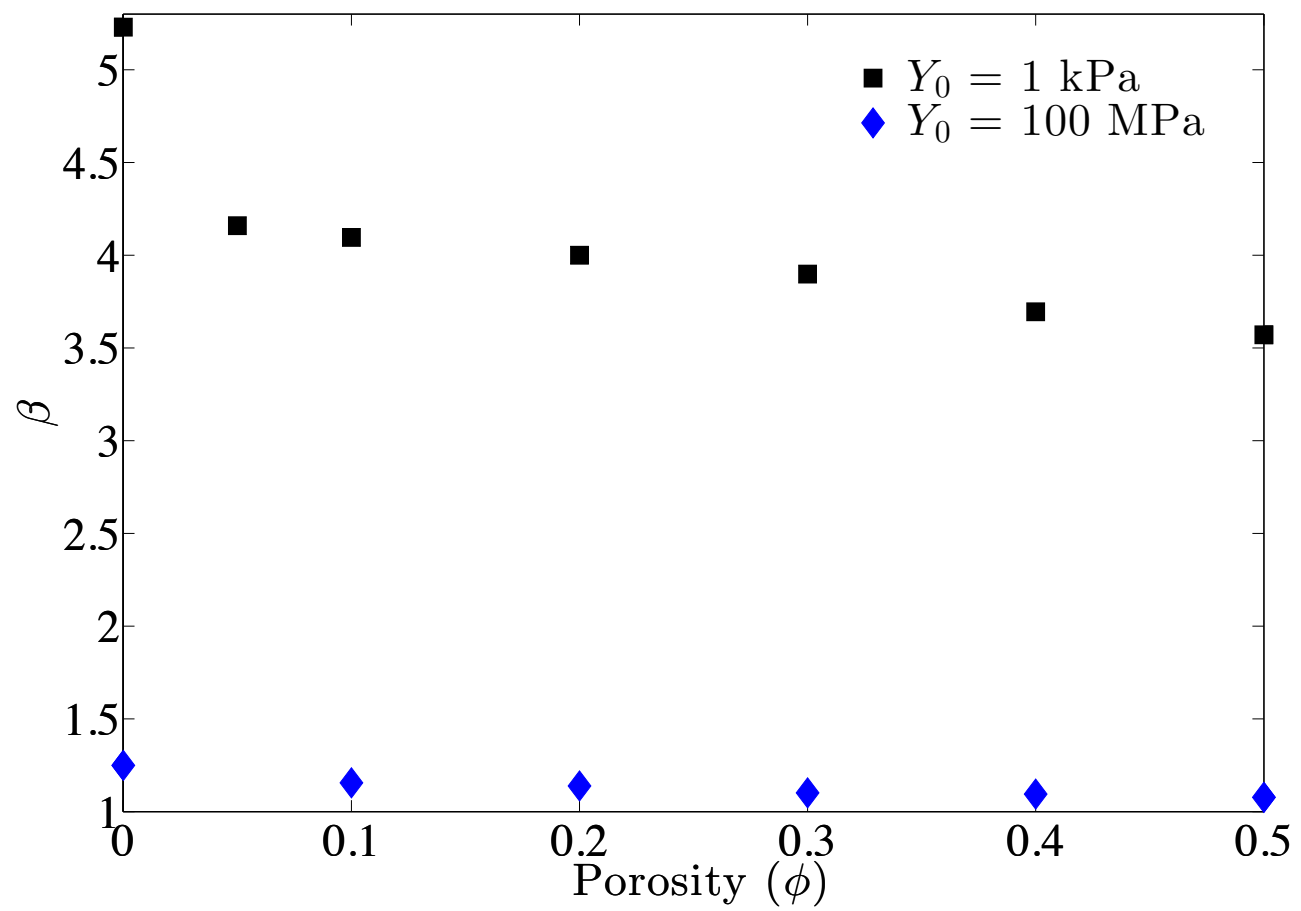

Figure 4: The presence of microporosity decreases the total momentum of target material ejected during an impact, reducing $\beta$. The effects of varying asteroid porosity on delivered momentum impulse are shown for different values of cohesion: $Y_{0}=1 \mathrm{kPa}$ and $Y_{0}=100 \mathrm{MPa}$. For the low-cohesion cases, there is an abrupt decrease in $\beta$ between nonporous and slightly porous $(\phi=0.05)$ material and a more gradual decrease with further increasing microporosity. For the high-cohesion cases, porosity effects on momentum transfer are less prominent. Data from runs 12, 14-24 (Table 2).

helpful for achieving larger deflection velocities. In the case shown, the impacts are near the disruption limit of a 100-m asteroid, so that more significant porosity also results in deflection velocities nearer to the asteroid's escape speed. While porosity will help reduce the risk of disruption through attenuation of the impact-generated shock wave, when the required $\Delta v$ is near to an asteroid's escape speed, another mitigation method may be required.

The risk of disruption can be assessed by tracking the post-impact center-of-mass velocity, $v_{\mathrm{COM}}$, and evaluating the fraction of asteroid material (other than ejecta) for which the speed difference, $\|\delta v\|=\left\|v_{\mathrm{COM}}-v\right\|$ exceeds escape speed. The disrupted fraction of 100$\mathrm{m}$ asteroids with different initial porosities is shown in Figure 6. Even a small amount of porosity lowers disruption risk; cases with $\phi=0.1-0.5$ approach a disrupted fraction of $\sim$ 0.003, while a much larger fraction (0.184) of the non-porous case is disrupted at $0.1 \mathrm{~s}$ after 


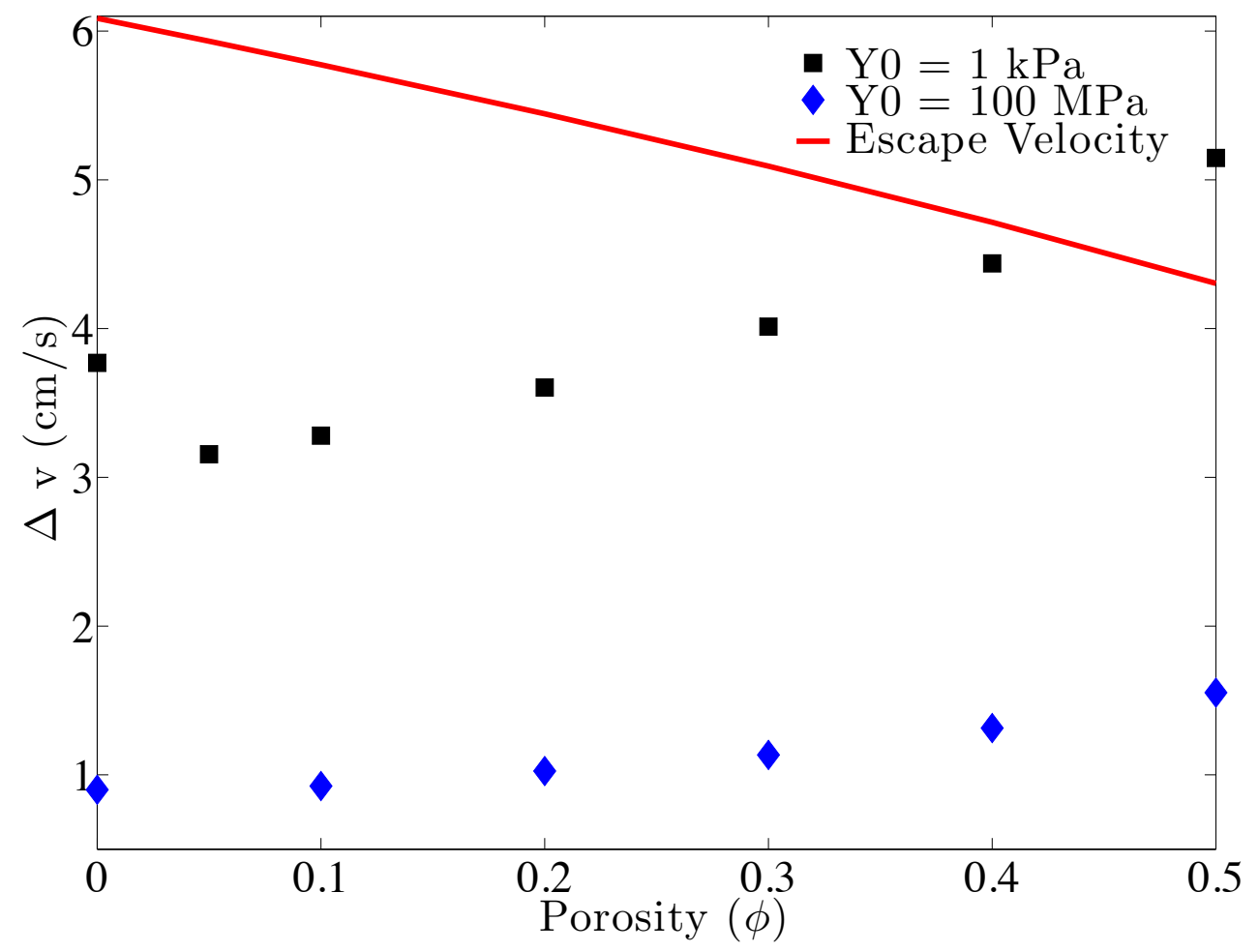

Figure 5: The final change in velocity, $\Delta v$, for $100-\mathrm{m}$ asteroids of different porosities with low $(1 \mathrm{kPa})$ and high (100 MPa) cohesion. Asteroid escape speed is plotted for reference. As porosity increases, total target mass decreases, producing larger deflection velocities for a given momentum impulse. This effect dominates over the decrease in $\beta$ with increasing porosity (Fig. 4), causing more porous asteroids to receive larger changes in velocity.

impact.

\subsection{Rotation}

Pre-impact spin periods of 100 seconds and 2.5 hours were used to assess rotational effects on both momentum delivery and disruption risk. These spin rates correspond to some of the

274 fastest known rotators $(P=100 \mathrm{~s})$ and to rotators just above the 2.2 hour "spin limit" for a cohesionless gravitational aggregate (Statler et al., 2013; Pravec and Harris, 2000). Impact locations were placed at the equator, to maximize rotational effects on ejecta velocities. In both cases, rotation did not significantly affect the final $\beta$ value calculated for the asteroids.

278 Results for the time evolution of $\beta$ for the $P=100 \mathrm{~s}$ asteroids are plotted in Figure 7 . In both 279 the strengthless and with-strength cases, the difference in calculated $\beta$ values were $<2 \%$. 280 Similar results were found for the asteroids with $2.5 \mathrm{hr}$ rotation periods: including rotation 


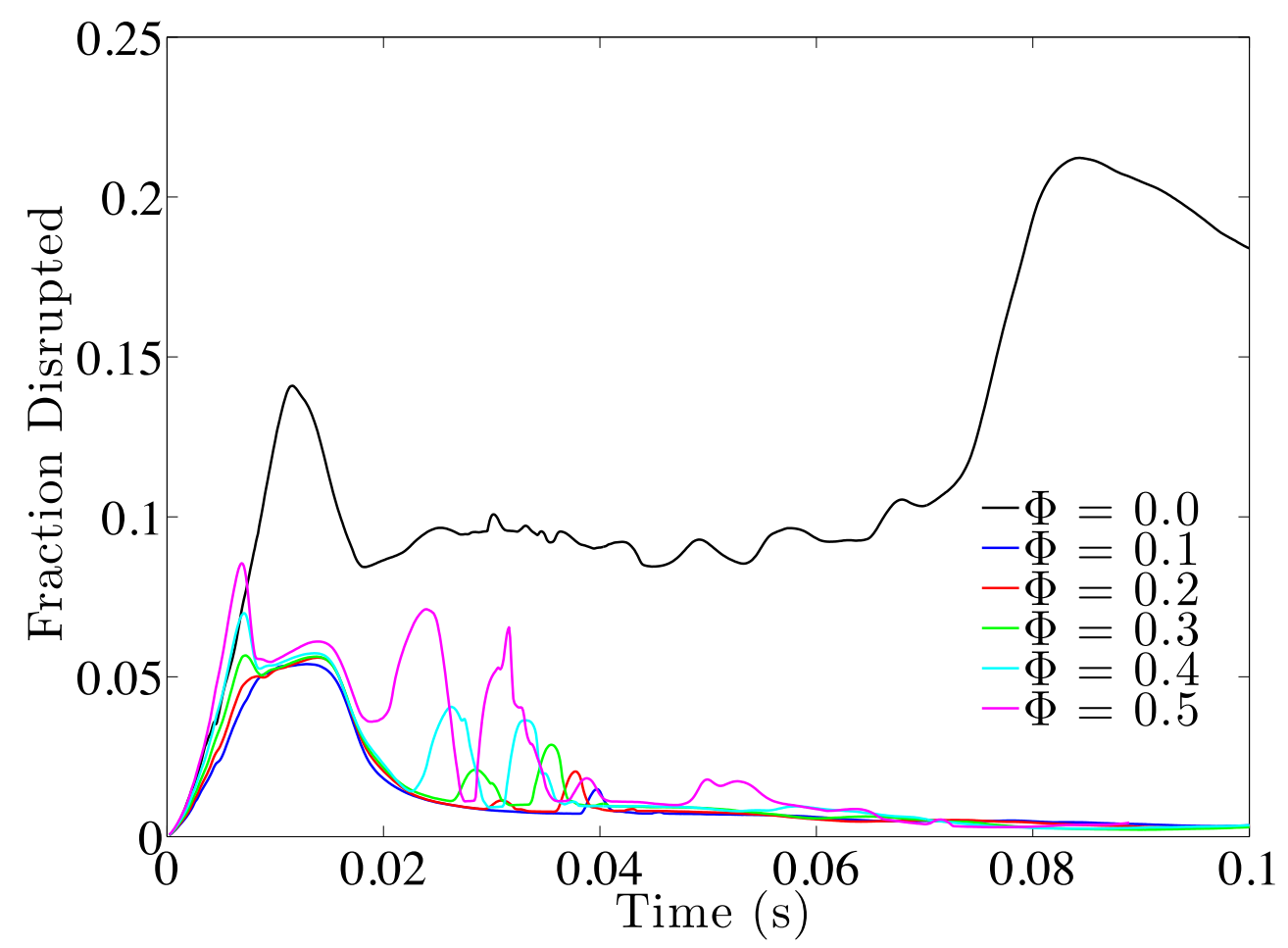

Figure 6: For kinetic-impact scenarios near the asteroid's disruption limit, tracking the fraction of nonejecta material that is mobilized above escape speed provides a measure of the disruption risk. For the high cohesion $\left(Y_{0}=100 \mathrm{MPa}\right)$ examples shown, even a small amount of porosity, $\phi$, is protective against disruption, lowering the fraction of disrupted mass from 0.184 to $\sim 0.003$ (at 0.1 s post-impact). Data is from runs 12, 20-24 (Table 2).

281 282

increased $\beta$ by only $0.16 \%$ (run numbers 27 and 28 in Table 2). As expected (and documented in Section 3.2), strength reduces the efficiency of momentum transfer for spinning and nonspinning asteroids. In order to search for maximal rotation-related perturbations, relatively low impact velocities $\left(v_{i}=5 \mathrm{~km} / \mathrm{s}\right)$ were used, so that the time scale of crater formation was nearer to the timescale of rotation.

In contrast, the risk of disruption is found to be affected by pre-impact rotation. Figure 8 shows non-rotating (a) and rotating (b) asteroids $(D=100 \mathrm{~m})$ impacted by $5000 \mathrm{~kg}$ masses at $5 \mathrm{~km} / \mathrm{s}$, at at time 0.12 seconds after impact. Blue color indicates material bound to the asteroid, while other colors represent material exceeding the asteroid's escape velocity. Material that is not part of the crater ejecta curtain but is mobilized above escape speed can contribute to unintentional disruption. Along the equator of the rotating asteroid, significant material appears to be dislodged by the impact. The fraction of target mass that 


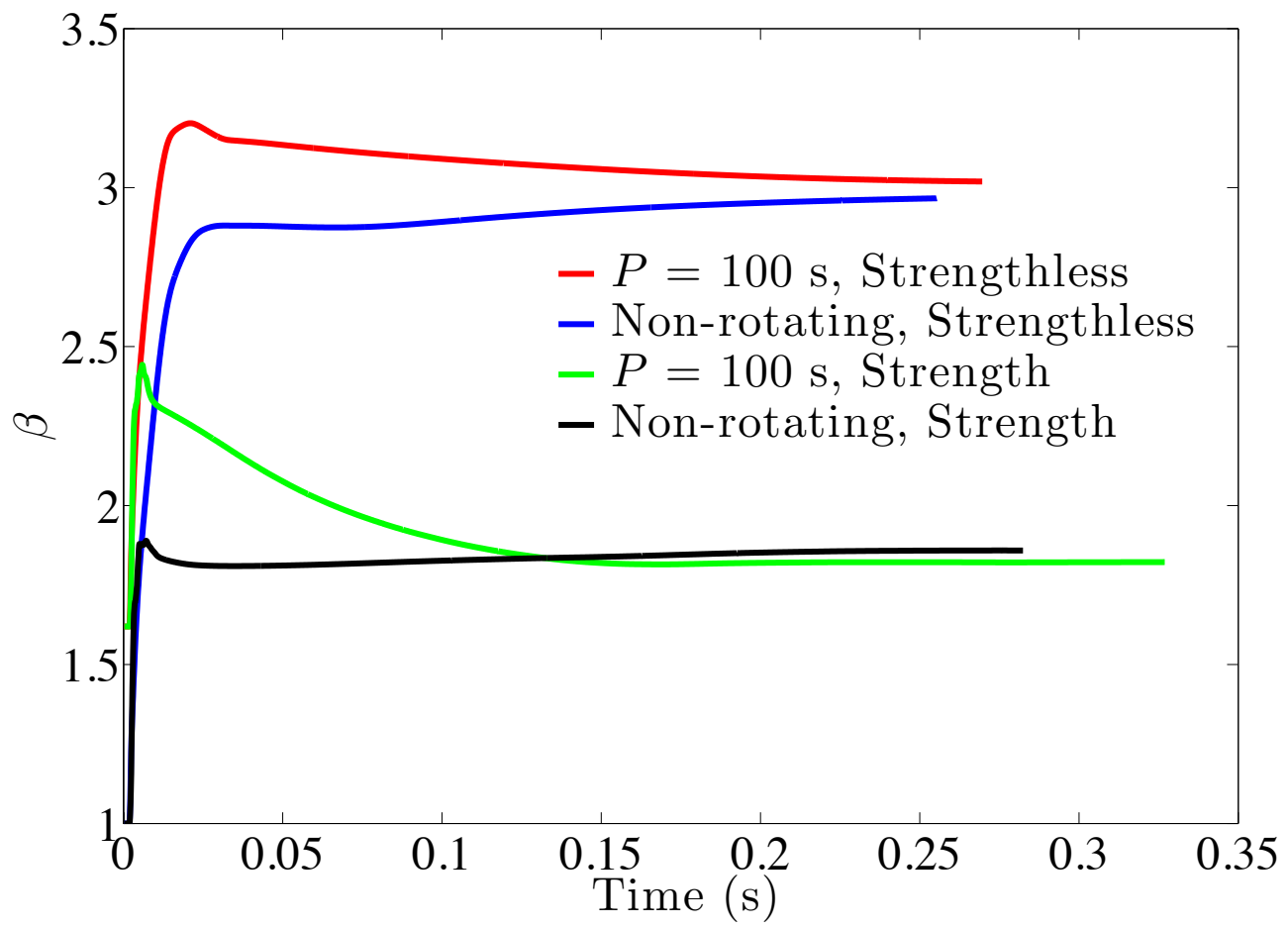

Figure 7: Time evolution of $\beta$ for a fast-rotating asteroid $(P=100 \mathrm{~s})$, with and without strength, compared to a non-rotating asteroid. While, at early times, the spinning asteroid appears to receive a slight enhancement in momentum transfer, both the rotating and non-rotating cases converge to the same $\beta$ values at later times. Results from runs 25-28 (Table 2).

will escape (apart from the desirable escape of material within the ejecta curtain), relative to the asteroid's center-of-mass velocity, is found to be 0.029 for the rotating case, a significant increase from the 0.007 found in the non-rotating example. In situations where the required $\Delta \mathrm{v}$ corresponds to impact conditions that approach an asteroid's disruption threshold, this enhanced disruption risk warrants extra caution, particularly for fast-rotating asteroids.

\subsection{Shape}

As an investigation into the effects of local asteroid topography on delivered momentum impulse, simulated impacts were carried out into asteroid Golevka, using a radar-derived shape model. Approximately $500 \mathrm{~m}$ across, Golevka represents the upper size range that a kinetic impactor could deflect off of an Earth-impacting orbit, even with decades of warning time (Dearborn and Miller, 2014). The asteroid's unusual shape, including many concave regions, serves as an interesting test case. Adequately resolving a 10,000-kg impactor while 


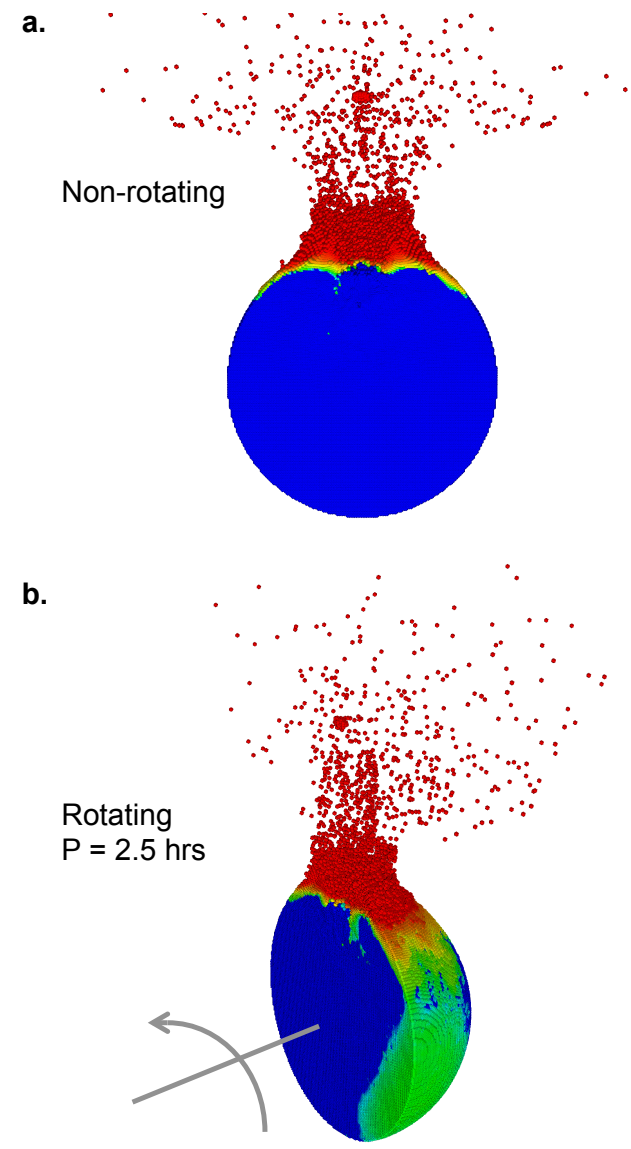

Figure 8: Rotational effects on the disruption risk to asteroids: top (a) depicts a non-rotating asteroid, while bottom (b) shows an asteroid rotating with a period of 2.5 hours. Blue material is below asteroid escape speed. The green regions along the equator of the rotating case represent asteroid material that has been unfavorably dislodged by the impact: it is not part of the crater ejecta curtain yet has been mobilized above escape speed. The fraction of unfavorably dislodged material is $2.9 \%$ for the rotating case, significantly more than the $0.7 \%$ calculated for the non-rotating case. Results from runs 29-30 (Table 2).

modeling an entire 500-m asteroid is computationally expensive, so the problem domain was confined to a region extending 80 meters from the chosen impact site.

The extent of damage produced by a $10 \mathrm{~km} / \mathrm{s}$ impact into asteroid Golevka (modeled as $20 \%$ porous $\mathrm{SiO}_{2}$ ) is shown in Figure 9. While the impactor collides along one of the asteroid's principal axes, the final direction of push is not through the asteroid's center of mass, as the ejecta momentum vector is directed 7 degrees off axis. Variations associated with local topography can thereby degrade the efficacy of the kinetic-impactor approach, leading to a smaller effective $\beta$ (where $\beta$ is defined as the momentum multiplication along 


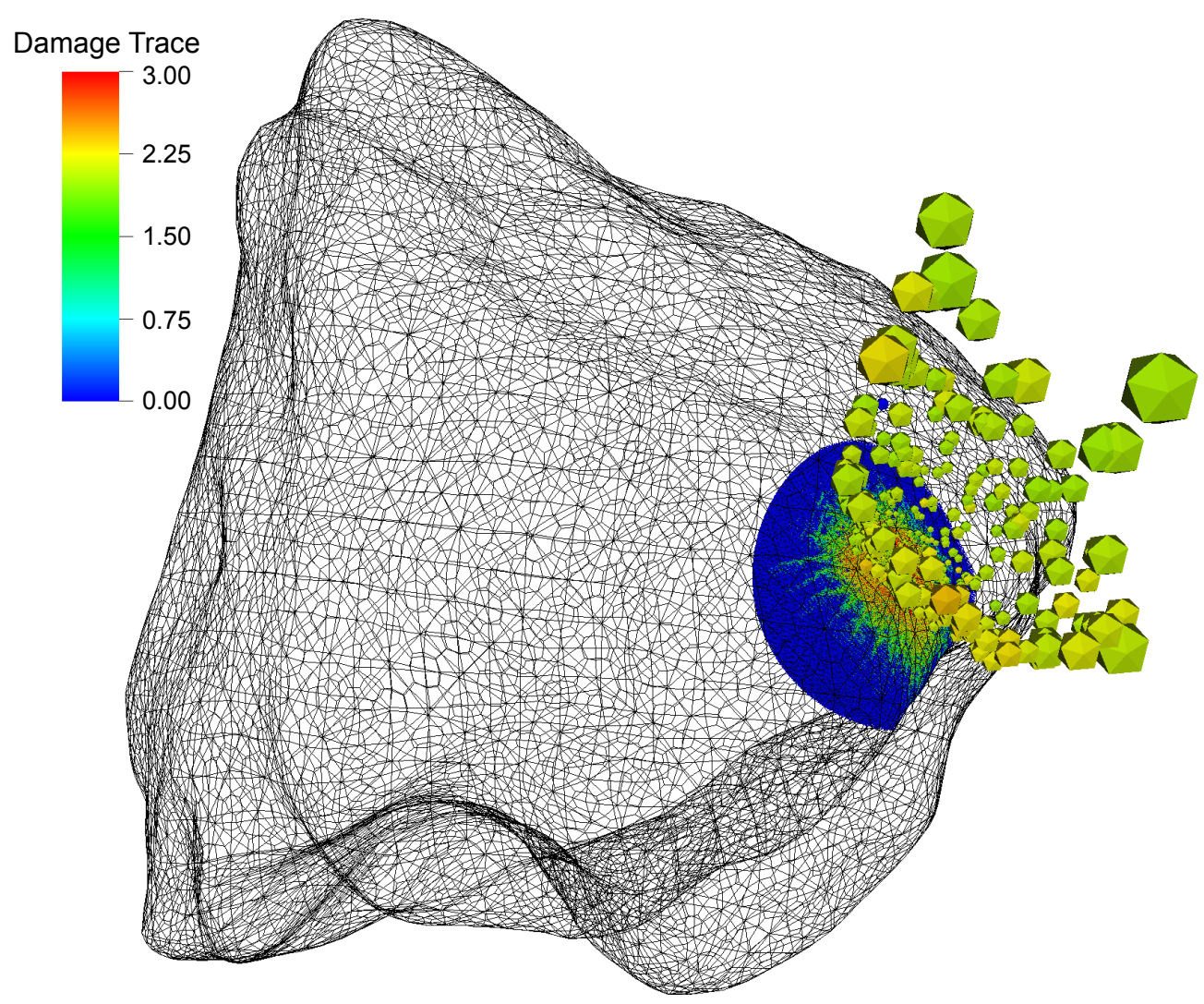

Figure 9: A shape model for asteroid Golevka is impacted by a 10,000-kg mass traveling at $10 \mathrm{~km} / \mathrm{s}$ along a principal axis of the asteroid. Colors denote the trace of the damage tensor, shown at $0.074 \mathrm{~s}$ after impact. The ejecta's momentum vector is directed approximately 7 degrees off axis from the impactor's trajectory. Asteroid topography can thus affect the resulting direction of push by a kinetic impactor, in practice introducing undesirable variation into the delivered momentum impulse. The final change in asteroid velocity for this example is $\sim 1 \mathrm{~mm} / \mathrm{s}$. The modeled region is confined to $80 \mathrm{~m}$ from the impact site (colored volume), while the entirety of the shape model is included for context.

313 the impact trajectory). In this case, the final $\beta$ calculated is 2.33 , which corresponds to a 314 modest change in velocity of $\Delta v \sim 1 \mathrm{~mm} / \mathrm{s}$.

\subsection{Velocity Scaling}

316 While a nominal impact velocity of $10 \mathrm{~km} / \mathrm{s}$ was employed for most simulations in this 317 study, we investigated the velocity scaling for momentum transfer, for comparison with ana318 lytical models and available experimental data (Holsapple and Housen, 2012). Experiments 319 are necessarily limited to smaller impact sizes and lower velocity regimes $(<6 \mathrm{~km} / \mathrm{s})$. Numerical modeling allows the full range of likely encounter velocities to be investigated at the 
impact-size regime of interest for asteroid deflection.

Based upon point-source scaling relations, the velocity scaling for the momentum of escaping ejecta, $P$, relative to impactor momentum, $m U$, should follow a power law:

$$
\frac{P}{m U}=\beta-1 \sim U^{3 \mu-1}
$$

where $\mu$ is the velocity-scaling exponent of the impact coupling parameter from point-source solutions (Holsapple and Schmidt, 1987; Holsapple and Housen, 2012). The value of $\mu$ is material dependent and may vary between the limits imposed by pure momentum scaling $(\mu=1 / 3)$ and pure energy scaling $(\mu=2 / 3)$. Experiments using geological materials find that $\mu$ generally ranges from 0.35 to 0.55 (Housen and Holsapple, 2011).

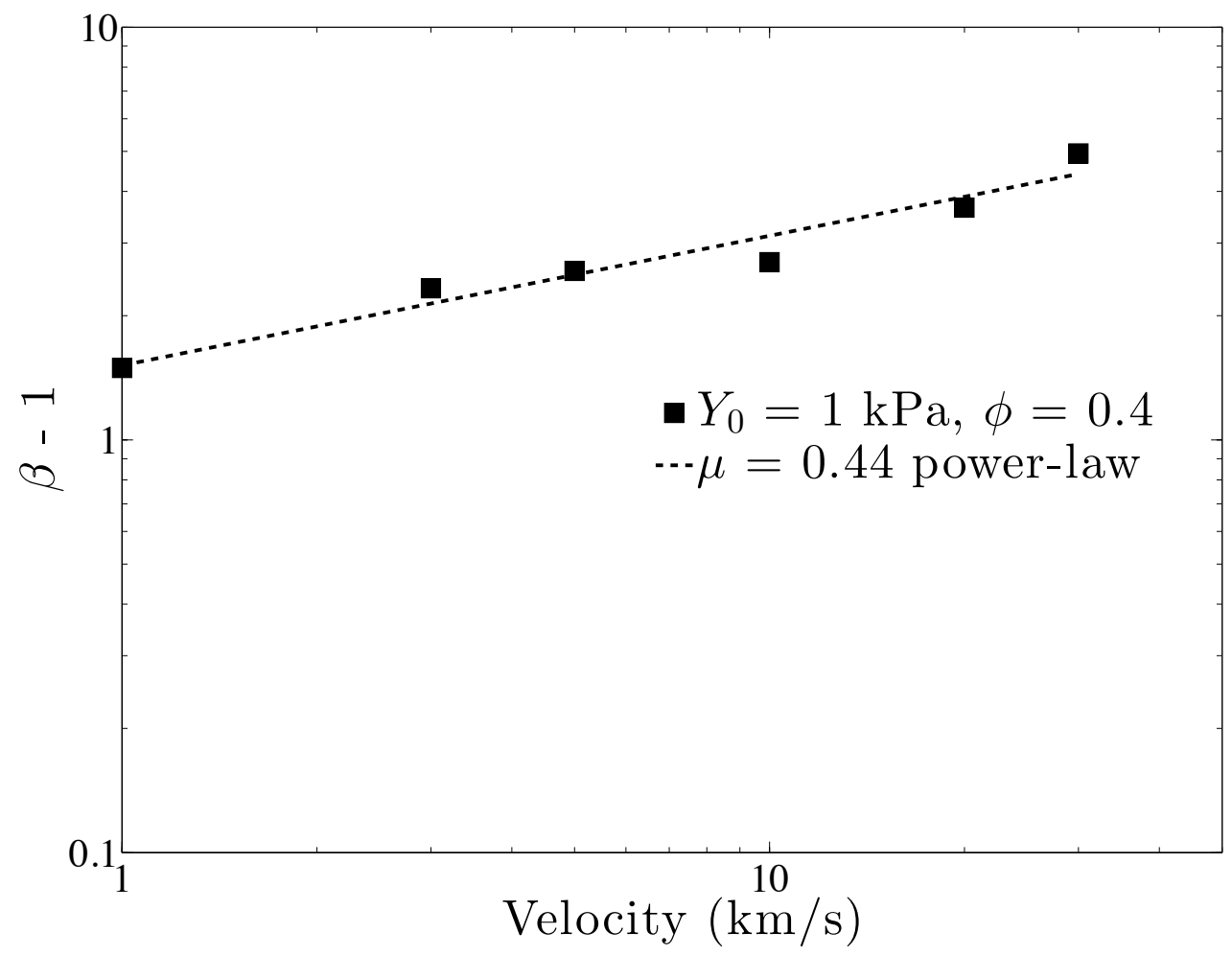

Figure 10: Velocity scaling of $\beta$ for $1-30 \mathrm{~km} / \mathrm{s}$ impacts into $40 \%$ porous, low-cohesion $\left(Y_{0}=1 \mathrm{kPa}\right)$ asteroid targets $(D=100 \mathrm{~m})$. Momentum delivery is approximated by a power-law slope of $0.39(\mu=0.44)$. This value of $\mu$ is consistent with impact experiments that exhibit values of $\mu$ ranging from 0.35 (perlite-sand mixture) to 0.55 (competent rock) (Housen and Holsapple, 2011). Results from runs 6, 31-35 (Table 2).

Results from impact velocities ranging from $1 \mathrm{~km} / \mathrm{s}$ to $30 \mathrm{~km} / \mathrm{s}$ are plotted in Figure 10.

330 This set of simulations used low-cohesion $\left(Y_{0}=1 \mathrm{kPa}\right)$ and $40 \%$ porous material. A power- 


\section{4. Discussion}

law fit to the data yields $\mu=0.44$, intermediate between experimentally determined values of $\mu$ for sand and competent rock (Holsapple, 1993; Housen and Holsapple, 2011). Although prior numerical study by Jutzi and Michel (2014) found impacts $5-15 \mathrm{~km} / \mathrm{s}$ to be described by $\mu=0.62$, closer to pure energy scaling, this difference is partly attributed to the higher value of cohesion used in that study, $Y_{0}=100 \mathrm{MPa}$, representative of relatively strong rock. The weaker material used to derive $\mu$ in the present work more closely approximates the idealized, "perfectly porous" case described in Holsapple and Schmidt (1987), for which $\mu$ is predicted to be closer to momentum scaling $(\mu=0.37-0.40)$. Differing values for numericallycomputed $\mu$ may also arise from other subtle differences in the modeled material properties, including the internal friction coefficients used and the yield strength at large pressures.

\subsection{Numerical Resolution}

For under-resolved simulations, as numerical resolution increases, the velocity change imparted to an asteroid by a kinetic impact decreases (Owen, 2014) and the extent of damaged target material also decreases (Owen et al., 2015). Simulations at a range of resolutions are necessary to determine under what conditions the results exhibit numerical convergence. To investigate resolution effects, we modeled a region extending 20 meters from the impact point on a 100-meter asteroid - confining the problem to this domain size allowed the highestresolution simulations to be run on 512 processors or less. The changing damage patterns associated with higher-resolution models are shown in Figure 11. As the damaged region decreases in size, less mass is ejected above escape speed and $\beta$ decreases. Resolution effects on the $\beta$ value calculated for $5 \mathrm{~km} / \mathrm{s}$ impacts are shown in Figure 12, exhibiting convergence at a spatial resolution of $15 \mathrm{~cm}$. The lowest-resolution case $(44.5 \mathrm{~cm})$ overestimates $\beta$ by approximately $12.25 \%$. As most calculations reported in this study modeled the entire asteroid, some required somewhat lower resolution than the optimal $15 \mathrm{~cm}$. The approximate errors in $\beta$ attributed to resolution effects are noted in Table 2.

The results presented demonstrate the wide breadth of asteroid characteristics affecting the impulse delivered to an asteroid by a kinetic deflection, even within the bounds of a 

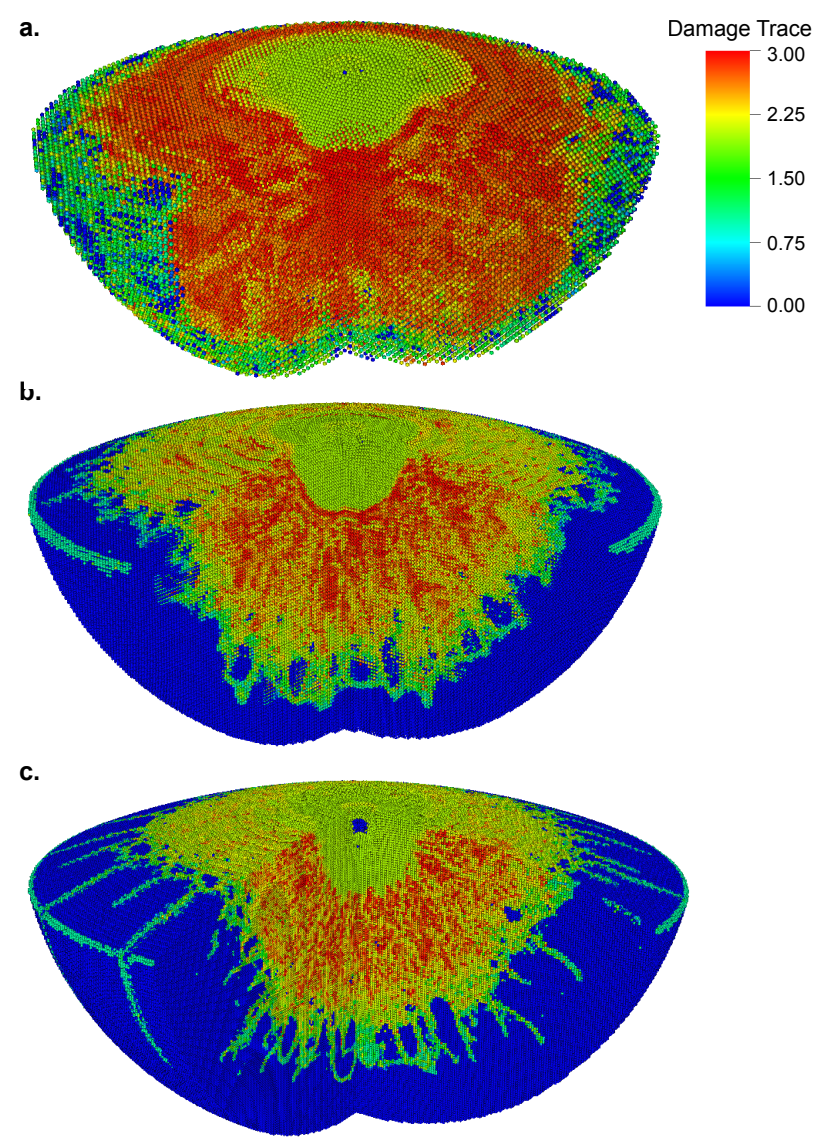

Figure 11: Increasing numerical resolution limits the extent of damage in kinetic-impact simulations: a. 44.5 $\mathrm{cm}$, b. $22.25 \mathrm{~cm}$, c. $14.83 \mathrm{~cm}$. The trace of the damage tensor is plotted 0.05 seconds after a $5 \mathrm{~km} / \mathrm{s}$ impact, after crack propagation has ceased for the two higher-resolution cases. The problem domain is confined to 20 meters from the impact site on a 100-meter asteroid to permit the calculation to be run on 512 processors or less. A quadrant is cut away from each image, so that damage accumulated internally to the asteroid can be visualized. Models employed $40 \%$ porous $\mathrm{SiO}_{2}, Y_{0}=100 \mathrm{MPa}$, and 1000-kg impactors.

uniform $\mathrm{SiO}_{2}$ composition. While these calculations are not exhaustive for the range of initial conditions describing real near-Earth asteroids, they provide baseline information on relations between some of the most fundamental, unconstrained parameters and an asteroid's response.

Porosity is well known to dampen momentum transfer by impacts, both from exper364 imental work (Holsapple and Housen, 2012) and numerical study (Asphaug et al., 1998; 365 Jutzi and Michel, 2014). The present work has explored the trends associated with in366 creasing porosity, under varied assumptions about the cohesion of asteroid-target material. 


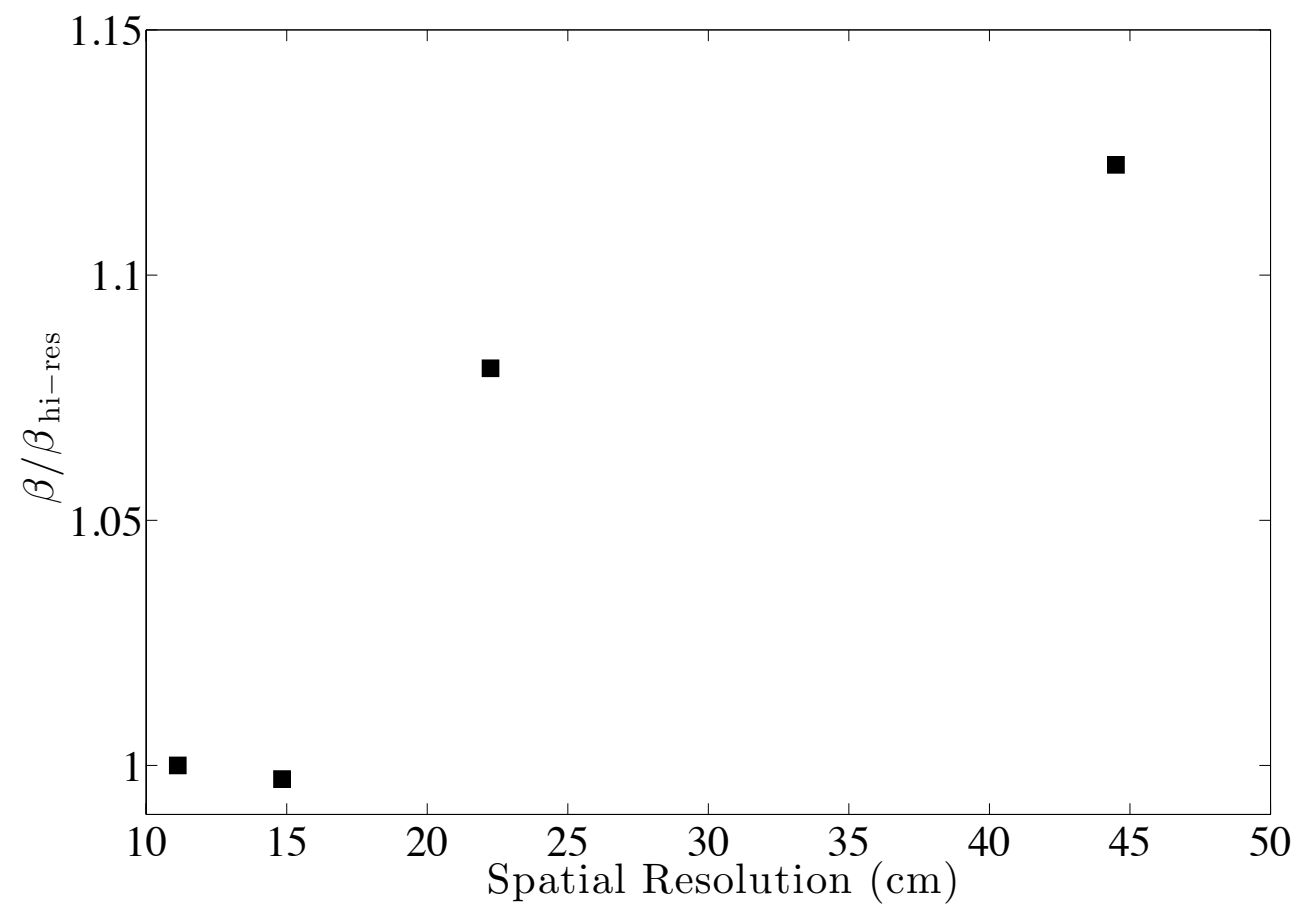

Figure 12: A graph of $\beta$, normalized by the highest-resolution result, as a function of numerical resolution (resolutions shown: 11.1, 14.8, 22.3, $44.5 \mathrm{~cm}$ ). Convergence is achieved near 15-cm resolution; the lowestresolution case over-predicts momentum-transfer efficiency by $12.25 \%$. Results are for the impact conditions described in the caption of Figure 11.

As expected, $\beta$ decreases with increasing porosity (Fig. 4). More interestingly, deflection velocities for increasingly porous asteroids are found to be greater, due to decreased asteroid mass for fixed asteroid size (Fig. 5). Additionally, even moderate porosity is found to be helpful for preventing unintentional disruption of an asteroid (Fig. 6). Assessment of porosity effects was limited to the assumption of microporosity, using a strain-based $\epsilon-\alpha$ model (Wünnemann et al., 2006); previous deflection calculations by Jutzi and Michel (2014) used the $P-\alpha$ porosity model (Herrmann, 1969). Ongoing development of microporosity models will enable future comparisons between approaches that account for the compaction of porous geological materials in different ways. For example, the microporosity model described in Herbold et al. (2015) was developed specifically for asteroidal materials and includes poroelastic compaction, comminution of grains, and bulking. This thermomechanical constitutive model fully couples solid equation-of-state data for chondrites, porosity, yield surface, breakage/damage, and bulking response, so that each of these evolving variables are 
380

381

interdependent throughout the simulation. Modeling porosity and strength/damage in such a manner benefits from improved thermodynamic consistency.

The high porosities inferred for most asteroids may be partially distributed in macroporous structures, including large voids between more competent boulders or fragments from previous collisions and subsequent gravitational reaccumulation. Investigation of kineticimpact deflection for asteroids with void space distributed using either a combination of micro- and macro-porosity, or only microporosity, has so far found minimal difference between the two scenarios (Jutzi and Michel, 2014). However, the effects of structural inhomogeneities, including larger voids and fragments of varying compositions and competency, remain to be studied in detail. The problem is intrinsically linked to asteroid-strength effects, as the constitutive models governing various possible structural components (e.g., granular regolith, crystalline rock fragments) are necessarily variable. The importance of internal structure in determining asteroid-mitigation outcomes is likely greater for situations when deflection is not a workable option (short warning times) but, rather, robust disruption and dispersal of the fragments is required for the mass to miss the Earth (Dearborn and Miller, 2014). The effects of asteroid structure on the outcomes of various mitigation options will be explored in detail in future calculations.

Rotation rates among the population of smaller asteroids $(D<150 \mathrm{~m})$ can be particularly fast. During the past two decades, the number of known asteroids that rotate at periods less than $\sim 2.2$ hrs, has significantly increased (Harris, 1996; Pravec and Harris, 2000; Statler et al., 2013). Such spin rates indicate that some amount of cohesion is necessary to prevent disaggregation, although the amount of cohesion required may be small $(\sim 0.1-100 \mathrm{kPa})$ in many cases (Holsapple, 2007; Rozitis et al., 2014). Recent optical light-curve surveys indicate that rotation rates are strongly correlated with size — while nearly all asteroids smaller than $60 \mathrm{~m}$ are categorized as fast rotators, very few bodies larger than $170 \mathrm{~m}$ are fast-rotating (Statler et al., 2013). Since smaller asteroids are least well-characterized by near-Earth asteroid surveys (Harris and D'Abramo, 2015), future detections of potentially hazardous asteroids will likely include greater numbers of small and fast-rotating bodies.

As a result, asteroid-mitigation measures should account for rotational effects on asteroid response. The work presented here does not find a significant increase in $\beta$, even for 
very fast $(P=100 \mathrm{~s})$ spin periods. This is attributable to characteristic ejecta velocities (those that significantly contribute to $\beta$ ) significantly exceeding the velocity introduced by rotation. However, spin remains important for assessing the boundary between deflection and unintentional disruption for cases when $\Delta v \sim v_{\text {esc }}$. Pre-impact rotation, particularly for weak, low-cohesion bodies, can increase the risk of generating a poorly-dispersed fragment field, depending on the size and velocity of the impactor. In cases when kinetic deflection creates such a fragment field, the end result could cause greater damage than electing to take no action. In situations where there is not sufficient warning time to gently deflect small, fast-rotating bodies without significant risk of undesired disruption, other, more energetic options can be examined, e.g., intentional, robust disruption and fragment dispersal (Dearborn and Miller, 2014).

Rotation can also affect the impact point of a kinetic-deflection mission and, consequently, the uncertainty in momentum impulse introduced by shape effects. Targeting a specific impact point, even for asteroids well characterized by radar measurements or spacecraft data, is complicated by the rotational state. Hence, there may be uncertainty regarding the local topography at the impact point, which can compromise the effectiveness of crater ejecta in boosting the momentum impulse (as shown numerically in Section 3.5). The importance of shape in controlling the momentum vector of impact ejecta, while briefly highlighted here, will be described more fully in another paper, including detailed comparisons to the analytic model of Scheeres et al. (2015).

Off-axis impacts, which will arise from imperfect targeting and are also influenced by asteroid shape and rotational state, represent an additional source of variability in asteroid response. The idealized scenario of a vertical impact through a spherical asteroid's center of mass, in the direction of intended deflection, represents a best possible case for maximizing the effective $\beta$. Any deviation from this scenario will result in an oblique impact, decreasing the delivered $\Delta \mathrm{v}$ as ejecta momentum is directed downrange (Anderson et al., 2003, 2004). Furthermore, off-axis impacts introduce perturbations to the asteroid's spin state. A significant percentage $(\sim 9 \%)$ of near-Earth asteroids can be described as contact binaries (Benner et al., 2006), while many more exhibit elongated geometries. In these cases, it may be particularly important to account for impact targeting variability — for instance, shock 
wave interaction with the narrow, low-cohesion "neck" of a contact binary has been shown to affect both the final $\Delta v$ and the disruption of the impacted lobe (Asphaug et al., 1998). More simulations are needed to fully evaluate how $\beta$ decreases with increasingly oblique impacts and to evaluate the range of outcomes for off-axis impacts on non-spherical asteroids; this work is reserved for future contributions.

Impact velocity strongly influences asteroid response to kinetic deflections and may vary considerably for different asteroid-intercept scenarios. It is therefore essential to have velocity scaling relationships for impacts into a variety of target types. Laboratory measurements have provided important data on the velocity scaling of $\beta$ for different geological materials at somewhat lower velocities $(<6 \mathrm{~km} / \mathrm{s}$ ) (Holsapple and Housen, 2012), while numerical simulations extend experimentally determined power laws to the higher possible impact velocities for deflection missions (up to approximately $30 \mathrm{~km} / \mathrm{s}$ ). The calculations presented here are the first to probe the full range of laboratory-to-deflection mission-relevant velocities (1- 30 $\mathrm{km} / \mathrm{s}$ ) and find that the power law describing the simulation results is consistent with available experimental data (Holsapple and Housen, 2012). While the velocity scaling exponent $\mu$ will vary between different target types, our calculated value of $\mu=0.44$ (for porous, lowstrength targets) provides a reasonable starting place for extrapolating momentum impulse delivery to a range of encounter velocities, using Equation 3.

Lastly, demonstrating numerical convergence is found to be particularly important for calculations of $\beta$ - under-resolved simulations will yield $\beta$ values that are too large. Modeling entire asteroids at a resolution sufficient to avoid over-estimating momentum transfer can be challenging. An alternative approach is to model a portion of the asteroid at higher resolution. Depending on the scenario, such a problem set-up may or may not be adequate. For example, when assessing the risk of disruption or large-scale damage and spallation across the asteroid, it is necessary to model the entire body. Impacts of higher velocities and larger masses can require larger domains (all or most of the asteroid) to avoid edge effects. In either case, the effects of varying the domain size and increasing resolution should be explored. Other strategies may include the use of finer-mass-resolution particles near the impact site and coarser masses at greater radial distances. However, this requires use of special node generators and must be thoroughly tested, to rule out introduction of error 
from the variable node masses.

The dependence of damage extent and total momentum of ejecta on the node-mass resolution also has implications for other asteroid-impact problems of interest. For example, the generation of collisional asteroid families by catastrophic disruption events (Michel et al., 2001, 2002, 2003, 2004) and calculations of the critical specific energy, $Q_{D}^{*}$, to disperse small bodies in both the strength- and gravity-dominated regimes (Asphaug et al., 1998; Benz and Asphaug, 1999; Leinhardt and Stewart, 2009) both rely upon SPH-type codes to determine the final fragment distribution after the impact phase. Sensitivity of the damage accumulation and ejected mass to numerical resolution may be an important consideration for future extensions of such studies.

\section{Conclusions}

Kinetic impactors represent a relatively simple and mature technology useful for deflecting asteroids when warning times are not too short and the bodies are not too large. However, details of the method's efficacy depend on a number of target parameters that may or may not be well-characterized before a deflection mission is staged, including composition, strength, porosity, rotational state, shape, and structure. It should be noted that these same asteroid characteristics are of interest for determining the outcomes of natural hypervelocity collisions and for understanding collisional histories.

We have systematically varied several asteroid properties expected to affect momentum delivery by high speed impacts, using an Adaptive Smoothed Particle Hydrodynamics code to model a range of deflection scenarios. Choice of equation of state, strength and damage models, porosity, rotation rate, and shape are all found to affect asteroid response. The momentum multiplier factor, $\beta$, decreases with increasing asteroid cohesion or with increasing porosity. For target bodies of a given size, larger porosity values actually result in greater deflection velocities, despite the decreased $\beta$ values, as a result of decreased asteroid mass. Calculations also reveal how porosity helps to mitigate disruption risk. Rotation, for the range of spin rates explored $(P=100-9000 \mathrm{~s})$, was not found to significantly affect momentum transfer but does increase the risk of unintentional disruption. Local asteroid topography at the impact point affects ejecta-momentum-vector direction, decreasing the 


\section{Acknowledgments}

effective $\beta$ calculated. final state of the asteroid.

Velocity scaling of the momentum delivered to asteroids was found to follow a power law described by $\mu=0.44$, consistent with the range of values determined by laboratory-scale impact experiments into geological materials $(0.35<\mu<0.55)$. Impact velocities spanned 1-30 km/s, covering the regimes explored by laboratory work as well as the upper limits of likely kinetic-deflection velocities. Our results provide a basis for extrapolating expected momentum multiplication factors under a variety of asteroid intercept conditions, a useful consideration for kinetic deflection mission design.

The results described here (1) highlight the importance of continued research to characterize the properties of near-Earth asteroids and (2) illustrate uncertainties in asteroid response that may remain before an actual kinetic deflection event. Although asteroids are diverse, gathering data on the strength and internal structure of just one or a few asteroids (through a combination of in-situ and remote-sensing investigations by future missions) would provide helpful information on some of the most poorly constrained geo-mechanical parameters. Uncertainties in topography at the impact point, introduced by the rotation and shape of individual asteroids, present complications for predicting the final momentum impulse. However, the statistical degradation of momentum transfer by shape effects can be accounted for during the planning stages of kinetic deflection missions. Opportunities to mount realistic tests of the kinetic-impactor concept, such as the AIDA mission, are exceedingly valuable, both for the new insight on an individual asteroid's material properties and for the experimental data for validation and verification of models within the impact-size ranges of interest to planetary defense. In such experiments, observables are not limited to $\beta$ - crater shape and size, ejecta evolution, and other details of the impact event, in addition to pre-impact reconnaissance, can be used to sort out the various effects that influence the

The authors thank Erik Asphaug and an anonymous reviewer for their comments, which strengthened this paper. Part of this work was funded by the Laboratory Directed Research and Development Program at LLNL under project tracking code 12-ERD-005, performed 
A’Hearn, M.F., Belton, M.J.S., Delamere, W.A., Kissel, J., Klaasen, K.P., McFadden, L.A., Meech, K.J., Melosh, H.J., Schultz, P.H., Sunshine, J.M., Thomas, P.C., Veverka, J., Yeomans, D.K., Baca, M.W., Busko, I., Crockett, C.J., Collins, S.M., Desnoyer, M., Eberhardy, C.A., Ernst, C.M., Farnham, T.L., Feaga, L., Groussin, O., Hampton, D., Ipatov, S.I., Li, J.Y., Lindler, D., Lisse, C.M., Mastrodemos, N., Owen, W.M., Richardson, J.E., Wellnitz, D.D., White, R.L., 2005. Deep Impact: Excavating Comet Tempel 1. Science 310, 258-264.

Anderson, J.L.B., Schultz, P.H., Heineck, J.T., 2003. Asymmetry of ejecta flow during oblique impacts using three-dimensional particle image velocimetry. Journal of Geophysical Research (Planets) 108, 13-1.

Anderson, J.L.B., Schultz, P.H., Heineck, J.T., 2004. Experimental ejection angles for oblique impacts: Implications for the subsurface flow-field. Meteoritics and Planetary Science 39, 303-320.

Asphaug, E., Collins, G., Jutzi, M., 2015. Global Scale Impacts, in: Michel, P., DeMeo, F.E., Bottke, W.F. (Eds.), Asteroids IV, University of Arizona Press.

Asphaug, E., Ostro, S.J., Hudson, R.S., Scheeres, D.J., Benz, W., 1998. Disruption of kilometre-sized asteroids by energetic collisions. Nature 393, 437-440.

Benner, L.A.M., Nolan, M.C., Ostro, S.J., Giorgini, J.D., Pray, D.P., Harris, A.W., Magri, C., Margot, J.L., 2006. Near-Earth Asteroid 2005 CR37: Radar images and photometry of a candidate contact binary. Icarus 182, 474-481.

Benz, W., Asphaug, E., 1994. Impact simulations with fracture. I. Method and tests. Icarus $107,98-116$.

Benz, W., Asphaug, E., 1995. Simulations of brittle solids using smooth particle hydrodynamics. Comput. Phys. Commun. 87, 253-265. 
Benz, W., Asphaug, E., 1999. Catastrophic Disruptions Revisited. Icarus 142, 5-20.

Benz, W., Cameron, A.G.W., Melosh, H.J., 1989. The origin of the Moon and the single impact hypothesis. III. Icarus 81, 113-131.

Britt, D., Yeomans, D., Housen, K., Consolmagno, G., 2002. Asteroid density, porosity, and structure, in: Bottke, W.F., Cellino, A., Paolicchi, P., Binzel, R.P. (Eds.), Asteroids III, University of Arizona Press.

Britt, D.T., Consolmagno, G.J., 2000. The Porosity of Dark Meteorites and the Structure of Low-Albedo Asteroids. Icarus 146, 213-219.

Bruck Syal, M., Dearborn, D.S., Schultz, P.H., 2013. Limits on the use of nuclear explosives for asteroid deflection. Acta Astronaut. 90, 103-111.

Cheng, A., Atchison, J., Kantsiper, B., Rivkin, A., Stickle, A., Reed, C., Galvez, A., Carnelli, I., Michel, P., Ulamec, S., 2015. Asteroid impact \& deflection assessment mission. Acta Astronaut. 115, 262-269.

Collins, G.S., Melosh, H.J., Ivanov, B.A., 2004. Modeling damage and deformation in impact simulations. Meteoritics and Planetary Science 39, 217-231.

Cotto-Figueroa, D., Asphaug, E., Garvie, L., Morris, M., Rai, A., Chattopadhyay, A., Chawla, N., 2015. Scale-Dependent Measurements of Meteorite Strength and Fragmentation: Tamdakht (H5) and Allende (CV3), in: AAS/Division for Planetary Sciences Meeting Abstracts.

Dearborn, D.S.P., Miller, P.L., 2014. Deflecting or Disrupting a Threatening Object, in: Pelton, J.N., Allahdadi, F. (Eds.), Handbook of Cosmic Hazards and Planetary Defense, Springer.

Fritsch, F.N., 2011. LIP: The Livermore Interpolation Package, Version 1.4. LLNL-TR406719-REV-3 Lawrence Livermore National Laboratory, Livermore, CA.

Grady, D.E., Kipp, M.E., 1980. Continuum modelling of explosive fracture in oil shale. International Journal of Rock Mechanics and Mining Sciences 17, 147-157. 
Harris, A.W., 1996. The Rotation Rates of Very Small Asteroids: Evidence for 'Rubble Pile' Structure, in: Lunar and Planetary Science Conference, p. 493.

Harris, A.W., D'Abramo, G., 2015. The population of near-Earth asteroids. Icarus 257, $302-312$.

Herbold, E., Owen, J., Swift, D., Miller, P., 2015. Simulations of Defense Strategies for Bennu: Material Characterization and Impulse Delivery. Procedia Engineering 103, 173180.

Herrmann, W., 1969. Constitutive equation for the dynamic compaction of ductile porous materials. Journal of Applied Physics 40, 2490-2499.

Holsapple, K., Schmidt, R., 1987. Point source solutions and coupling parameters in cratering mechanics. J. Geophys. Res.-Sol. Ea. 92, 6350-6376.

Holsapple, K.A., 1993. The scaling of impact processes in planetary sciences. Annu. Rev. Earth Pl. Sc. 21, 333-373.

Holsapple, K.A., 2007. Spin limits of Solar System bodies: From the small fast-rotators to 2003 EL61. Icarus 187, 500-509.

Holsapple, K.A., Housen, K.R., 2012. Momentum transfer in asteroid impacts. I. Theory and scaling. Icarus $221,875-887$.

Housen, K.R., Holsapple, K.A., 2011. Ejecta from impact craters. Icarus 211, 856-875.

Howley, K., Managan, R., Wasem, J., 2014. Blow-off momentum from melt and vapor in nuclear deflection scenarios. Acta Astronaut. 103, 376-381.

Jutzi, M., 2015. SPH calculations of asteroid disruptions: The role of pressure dependent failure models. Planet. Space Sci. 107, 3-9.

Jutzi, M., Holsapple, K., Wünneman, K., Michel, P., 2015. Modeling asteroid collisions and impact processes, in: Michel, P., DeMeo, F.E., Bottke, W.F. (Eds.), Asteroids IV, University of Arizona Press. 
Jutzi, M., Michel, P., 2014. Hypervelocity impacts on asteroids and momentum transfer I. Numerical simulations using porous targets. Icarus 229, 247-253.

Kimberley, J., Ramesh, K.T., 2011. The dynamic strength of an ordinary chondrite. Meteorit. Planet. Sci. 46, 1653-1669.

Korycansky, D.G., Plesko, C.S., Jutzi, M., Asphaug, E., Colaprete, A., 2009. Predictions for the LCROSS mission. Meteoritics and Planetary Science 44, 603-620.

Kraus, R., Stewart, S., Swift, D., Bolme, C., Smith, R., Hamel, S., Hammel, B., Spaulding, D., Hicks, D., Eggert, J., et al., 2012. Shock vaporization of silica and the thermodynamics of planetary impact events. J. Geophys. Res.-Planet. 117.

Leinhardt, Z.M., Stewart, S.T., 2009. Full numerical simulations of catastrophic small body collisions. Icarus 199, 542-559.

Lindsay, S.S., Marchis, F., Emery, J.P., Enriquez, J.E., Assafin, M., 2015. Composition, mineralogy, and porosity of multiple asteroid systems from visible and near-infrared spectral data. Icarus 247, 53-70.

Lundborg, N., 1968. Strength of rock-like materials. International Journal of Rock Mechanics and Mining Sciences 5, 427-454.

Melosh, H., 2007. A hydrocode equation of state for $\mathrm{SiO}_{2}$. Meteorit. Planet. Sci. 42, 20792098.

Michel, P., Benz, W., Richardson, D.C., 2003. Disruption of fragmented parent bodies as the origin of asteroid families. Nature 421, 608-611.

Michel, P., Benz, W., Richardson, D.C., 2004. Catastrophic disruption of pre-shattered parent bodies. Icarus 168, 420-432.

Michel, P., Benz, W., Tanga, P., Richardson, D.C., 2001. Collisions and Gravitational Reaccumulation: Forming Asteroid Families and Satellites. Science 294, 1696-1700. 
Michel, P., Richardson, D.C., Durda, D.D., Jutzi, M., Asphaug, E., 2015. Collisional Formation and Modeling of Asteroid Families, in: Michel, P., DeMeo, F.E., Bottke, W.F. (Eds.), Asteroids IV, University of Arizona Press.

Michel, P., Tanga, P., Benz, W., Richardson, D.C., 2002. Formation of Asteroid Families by Catastrophic Disruption: Simulations with Fragmentation and Gravitational Reaccumulation. Icarus 160, 10-23.

Mitchell, J.K., Scott, R.F., Houston, W.N., Costes, N.C., Carrier, W.D., Bromwell, L.G., 1972. Mechanical Properties of Lunar Soil: Density, Cohesion, and Angle of Internal Friction, in: Lunar and Planetary Science Conference, p. 545.

Monaghan, J.J., 1992. Smoothed particle hydrodynamics. Annu. Rev. Astron. Astrophys. $30,543-574$.

National Research Council, 2010. Defending Planet Earth: Near-Earth-Object Surveys and Hazard Mitigation Strategies, National Academies Press, Washington, DC.

Owen, J.M., 2010. ASPH modeling of material damage and failure, in: Proceedings of the Fifth International SPHERIC Workshop, pp. 297-304.

Owen, J.M., 2014. A compatibly differenced total energy conserving form of sph. Int. J. Numer. Meth. Fl. 75, 749-774.

Owen, J.M., Miller, P., Rovny, J., Wasem, J., Howley, K., Herbold, E.B., 2015. Asteroid diversion considerations and comparisons of diversion techniques. Procedia Engineering $103,466-474$.

Owen, J.M., Villumsen, J.V., Shapiro, P.R., Martel, H., 1998. Adaptive Smoothed Particle Hydrodynamics: Methodology. II. Astrophys. J. Suppl. S. 116, 155-209.

Petrovic, J., 2001. Review mechanical properties of meteorites and their constituents. J. Mater. Sci. 36, 1579-1583.

Pravec, P., Harris, A.W., 2000. Fast and Slow Rotation of Asteroids. Icarus 148, 12-20. 
Rozitis, B., Maclennan, E., Emery, J.P., 2014. Cohesive forces prevent the rotational breakup of rubble-pile asteroid (29075) 1950 DA. Nature 512, 174-176.

Scheeres, D.J., McMahon, J.W., Jones, B.A., Doostan, A., 2015. Variation of delivered impulse as a function of asteroid shape, in: Aerospace Conference, 2015 IEEE, pp. 1-7.

Schultz, P.H., Eberhardy, C.A., Ernst, C.M., A'Hearn, M.F., Sunshine, J.M., Lisse, C.M., 2007. The Deep Impact oblique impact cratering experiment. Icarus 191, 84-122.

Sears, D., Sears, H.W., Ostrowski, D., Bryson, K., Dotson, J., Bruck Syal, M., Swift, D., 2015. A meteorite perspective on planetary defense. Planet. Space Sci. (under review).

Statler, T.S., Cotto-Figueroa, D., Riethmiller, D.A., Sweeney, K.M., 2013. Size matters: The rotation rates of small near-Earth asteroids. Icarus 225, 141-155.

Stickle, A., Atchison, J., Barnouin, O., Cheng, A., Crawford, D., Ernst, C., Fletcher, Z., Rivkin, A., 2015. Modeling momentum transfer from kinetic impacts: Implications for redirecting asteroids. Procedia Engineering 103, 577-584.

Thompson, S., Lauson, H., 1972. Improvements in the Chart D Radiation Hydrodynamic Code III: Revised Analytical Equation of State. SC-RR-710714 Sandia National Laboratory, Albuquerque, NM.

Tillotson, J.H., 1962. Metallic equations of state for hypervelocity impact. Technical Report. General Atomic Report GA-3216.

Wünnemann, K., Collins, G., Melosh, H., 2006. A strain-based porosity model for use in hydrocode simulations of impacts and implications for transient crater growth in porous targets. Icarus $180,514-527$. 
Table 1: Computational Parameters

\begin{tabular}{lr}
\hline Parameter & Value \\
\hline$\rho_{\text {target }}(\phi=0)$ & $2.65 \mathrm{~g} / \mathrm{cm}^{3}$ \\
$\rho_{\text {impactor }}$ & $2.70 \mathrm{~g} / \mathrm{cm}^{3}$ \\
$Y_{0}$ & $1 \mathrm{kPa}-100 \mathrm{MPa}$ \\
$Y_{m}$ & $1.5 \mathrm{GPa}$ \\
$\mu_{i}$ & 1.2 \\
$G$ & $22.7 \mathrm{GPa}$ \\
$m^{a}$ & 9.5 \\
$k^{a}$ & $8 \times 10^{37} \mathrm{~cm}^{-3}$ \\
$\phi$ & $0.0-0.5$ \\
$\epsilon_{e}$ & 0.0 \\
$\epsilon_{X}$ & -0.4 \\
$\kappa$ & 0.8 \\
\hline
\end{tabular}

${ }^{a}$ Weibull parameters from Jutzi (2015) 
Table 2: List of Kinetic Impact Simulations

\begin{tabular}{|c|c|c|c|c|c|c|c|c|c|}
\hline No. & $v_{i}{ }^{a}$ & $m_{i}^{b}$ & $D_{a}^{c}$ & $\operatorname{EOS}^{d}$ & $Y_{0}{ }^{e}$ & Porosity $^{f}$ & $P^{g}$ & Resolution $(\mathrm{cm})^{h}$ & $\beta$ \\
\hline 1 & 10 & 10 & 50 & $\mathrm{~T}$ & NS & 0.4 & - & 32.1 & 3.507 \\
\hline 2 & 10 & 10 & 50 & $\mathrm{~L}$ & NS & 0.4 & - & 32.1 & 3.087 \\
\hline 3 & 10 & 10 & 50 & A & NS & 0.4 & - & 32.1 & 2.898 \\
\hline 4 & 10 & 10 & 50 & $\mathrm{~T}$ & $\mathrm{VM}, 3.5 \mathrm{GPa}$ & 0.4 & - & 32.1 & 2.243 \\
\hline 5 & 10 & 1 & 100 & A & NS & 0.4 & - & 44.5 & 5.432 \\
\hline 6 & 10 & 1 & 100 & A & $1 \mathrm{kPa}$ & 0.4 & - & 44.5 & 3.695 \\
\hline 7 & 10 & 1 & 100 & $\mathrm{~A}$ & $10 \mathrm{kPa}$ & 0.4 & - & 44.5 & 3.693 \\
\hline 8 & 10 & 1 & 100 & A & $100 \mathrm{kPa}$ & 0.4 & - & 44.5 & 3.569 \\
\hline 9 & 10 & 1 & 100 & A & $1 \mathrm{MPa}$ & 0.4 & - & 44.5 & 1.917 \\
\hline 10 & 10 & 1 & 100 & A & $10 \mathrm{MPa}$ & 0.4 & - & 44.5 & 1.301 \\
\hline 11 & 10 & 1 & 100 & A & $50 \mathrm{MPa}$ & 0.4 & - & 44.5 & 1.196 \\
\hline 12 & 10 & 1 & 100 & A & $100 \mathrm{MPa}$ & 0.4 & - & 44.5 & 1.094 \\
\hline 13 & 10 & 1 & 100 & A & $\mathrm{VM}, 100 \mathrm{MPa}$ & 0.4 & - & 44.5 & 1.112 \\
\hline 14 & 10 & 1 & 100 & A & $1 \mathrm{kPa}$ & 0.0 & - & 44.5 & 5.230 \\
\hline 15 & 10 & 1 & 100 & A & $1 \mathrm{kPa}$ & 0.05 & - & 44.5 & 4.159 \\
\hline 16 & 10 & 1 & 100 & A & $1 \mathrm{kPa}$ & 0.1 & - & 44.5 & 4.096 \\
\hline 17 & 10 & 1 & 100 & A & $1 \mathrm{kPa}$ & 0.2 & - & 44.5 & 4.000 \\
\hline 18 & 10 & 1 & 100 & A & $1 \mathrm{kPa}$ & 0.3 & - & 44.5 & 3.898 \\
\hline 19 & 10 & 1 & 100 & A & $1 \mathrm{kPa}$ & 0.5 & - & 44.5 & 3.571 \\
\hline 20 & 10 & 1 & 100 & A & $100 \mathrm{MPa}$ & 0.0 & - & 44.5 & 1.249 \\
\hline 21 & 10 & 1 & 100 & A & $100 \mathrm{MPa}$ & 0.1 & - & 44.5 & 1.155 \\
\hline 22 & 10 & 1 & 100 & A & $100 \mathrm{MPa}$ & 0.2 & - & 44.5 & 1.138 \\
\hline 23 & 10 & 1 & 100 & A & $100 \mathrm{MPa}$ & 0.3 & - & 44.5 & 1.101 \\
\hline 24 & 10 & 1 & 100 & A & $100 \mathrm{MPa}$ & 0.5 & - & 44.5 & 1.077 \\
\hline 25 & 5 & 10 & 50 & $\mathrm{~T}$ & NS & 0.4 & 100 & 32.1 & 3.022 \\
\hline 26 & 5 & 10 & 50 & $\mathrm{~T}$ & NS & 0.4 & - & 32.1 & 2.965 \\
\hline 27 & 5 & 10 & 50 & $\mathrm{~T}$ & $\mathrm{VM}, 3.5 \mathrm{GPa}$ & 0.4 & 100 & 32.1 & 1.822 \\
\hline 28 & 5 & 10 & 50 & $\mathrm{~T}$ & $\mathrm{VM}, 3.5 \mathrm{GPa}$ & 0.4 & - & 32.1 & 1.858 \\
\hline 29 & 5 & 5 & 100 & $\mathrm{~L}$ & $100 \mathrm{MPa}$ & 0.4 & 9000 & 38.1 & 1.225 \\
\hline 30 & 5 & 5 & 100 & $\mathrm{~L}$ & $100 \mathrm{MPa}$ & 0.4 & - & 38.1 & 1.223 \\
\hline 31 & 1 & 1 & 100 & A & $1 \mathrm{kPa}$ & 0.4 & - & 44.5 & 2.494 \\
\hline 32 & 3 & 1 & 100 & A & $1 \mathrm{kPa}$ & 0.4 & - & 44.5 & 3.330 \\
\hline 33 & 5 & 1 & 100 & A & $1 \mathrm{kPa}$ & 0.4 & - & 44.5 & 3.567 \\
\hline 34 & 20 & 1 & 100 & A & $1 \mathrm{kPa}$ & 0.4 & - & 44.5 & 4.654 \\
\hline 35 & 30 & 1 & 100 & A & $1 \mathrm{kPa}$ & 0.4 & - & 44.5 & 5.945 \\
\hline
\end{tabular}

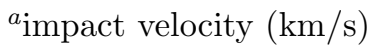

${ }^{b}$ impactor mass $\left(10^{3} \mathrm{~kg}\right)$

${ }^{c}$ asteroid diameter $(\mathrm{m})$

${ }^{d} \mathrm{~T}$ : Tillotson, L: LEOS, A: ANEOS

e Strength takes form of Equation 2, except NS: no strength, VM: von Mises

${ }^{f} \rho_{0}=2.65 \mathrm{~g} / \mathrm{cm}^{3}$

${ }^{g}$ Rotation Period (s)

${ }^{h}$ Convergence occurs near $15 \mathrm{~cm} ; 44.5$-cm resolution may over-estimate $\beta$ by $\sim 12.25 \%$ 